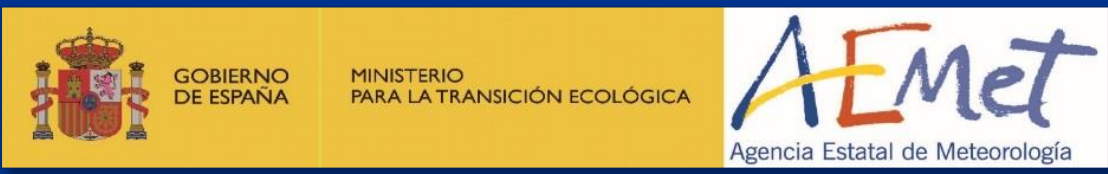

\title{
La cizalladura en el aeropuerto de Barcelona: episodio del 10 de septiembre de 2017
}

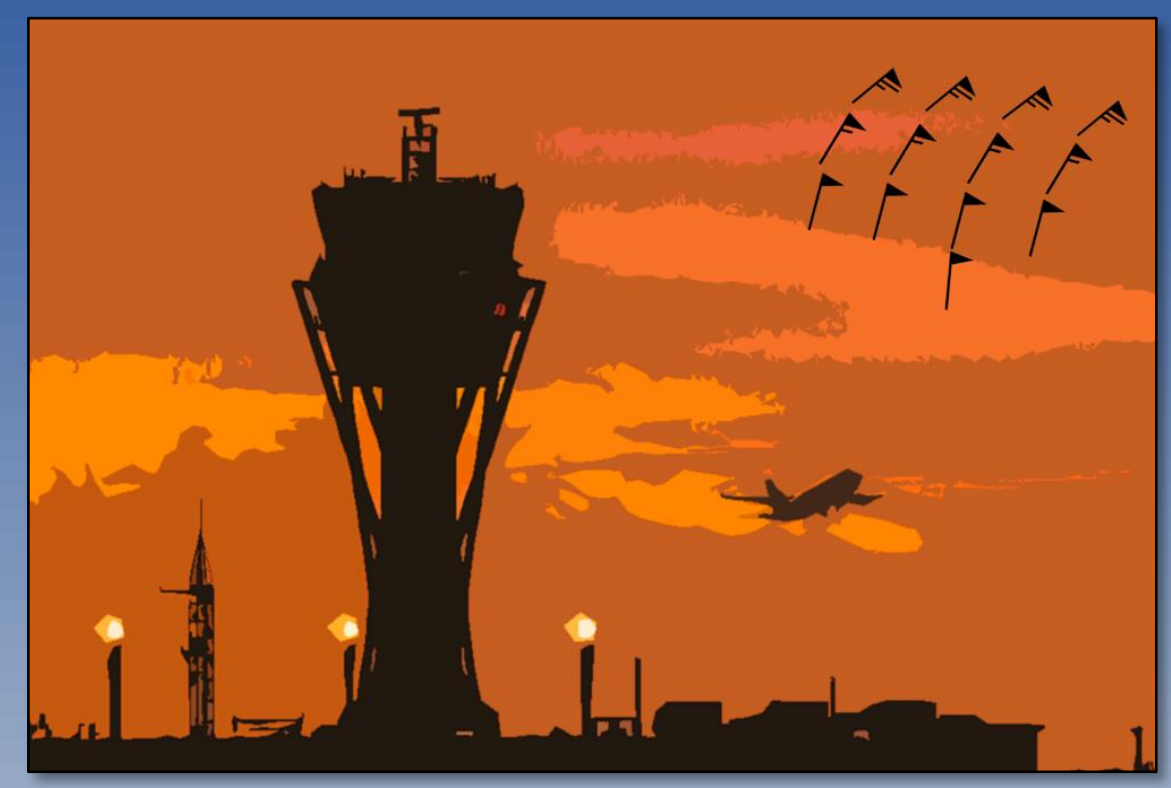

\section{Nota técnica 26 de AEMET}

Alejandro Méndez Frades, María Rosa Pons Reynés, Juan Simarro Grande, Inés Santos Atienza, Sergi González Herrero, Ramón Pascual Berghaenel, Agustí Pladevall Boix, Alfons Callado Pallarés y Marc Compte Rovirola. 
Aviso Legal: los contenidos de esta publicación podrán ser reutilizados, citando la fuente y la fecha, en su caso, de la última actualización.

Textos: Alejandro Méndez Frades, María Rosa Pons Reynés, Juan Simarro Grande, Inés Santos Atienza, Sergi González Herrero, Ramón Pascual Berghaenel, Agustí Pladevall Boix, Alfons Callado Pallarés y Marc Compte Rovirola.

\section{Edita:}

(C) Ministerio para la Transición Ecológica

Agencia Estatal de Meteorología

Unidad de Meteorología Aeronáutica

Madrid, 2019

Catálogo de Publicaciones de la Administración General del Estado:

http://publicacionesoficiales.boe.es/

NIPO: 639-19-002-X

https://doi.org//0.31978/639-19-002-X

Agencia Estatal de Meteorología (AEMET)

C/ Leonardo Prieto Castro, 8

28040 Madrid

http://www.aemet.es/

@Aemet_Esp

@Aemet_Cat

f https://www.facebook.com/AgenciaEstatalMeteorologia 


\section{Índice}

I. Resumen y objetivos.......................................................... 5

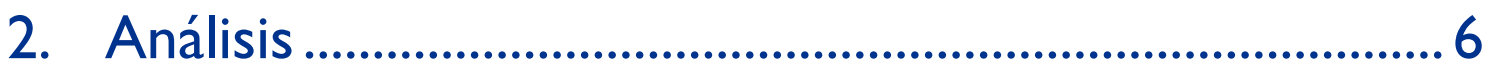

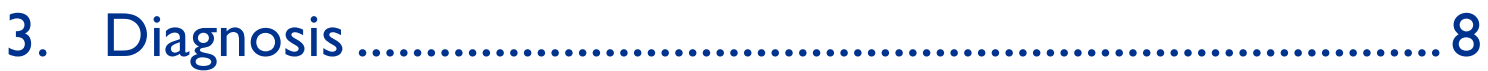

3.1. CARACTERIZACIÓN MESOESCALAR ............................................... 9

3.2. CARACTERIZACIÓN DEL ENTORNO MICROMETEOROLÓGICO..............11

3.3. MODELO CONCEPTUAL .................................................................. 12

4. Predicción.......................................................................

4.1. MODELO DE PREDICCIÓN DETERMINISTA HARMONIE-AROME

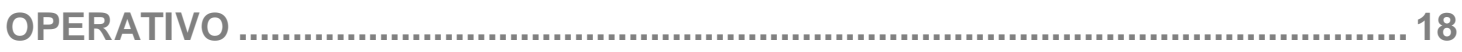

4.2. SECCIONES VERTICALES PREVISTAS POR HARMONIE-AROME

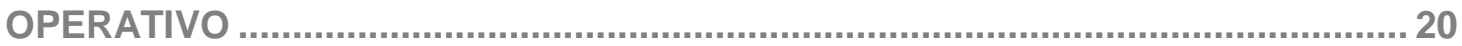

4.3. MODELO DE PREDICCIÓN DETERMINISTA HARMONIE-AROME 1 KM . 24

4.4. MODELO DE PREDICCIÓN PROBABILISTA AEMET-GSREPS .................. 25

4.5. PREDICCIÓN AERONÁUTICA .................................................... 27

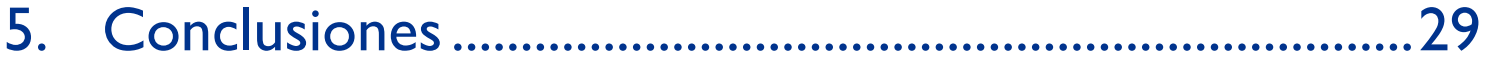

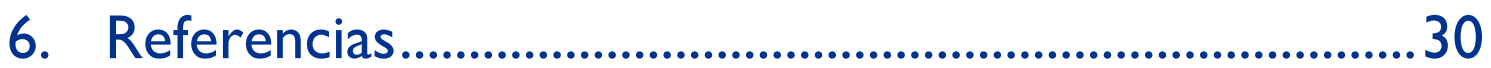

7. Acrónimos utilizados ......................................................3।

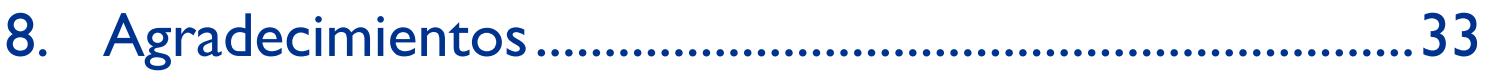




\section{Resumen y objetivos}

El estudio de este episodio se ha elaborado a fin de analizar con profundidad una situación operativa crítica que tuvo lugar en el aeropuerto internacional de BarcelonaEl Prat (en adelante, LEBL) el pasado 10 de septiembre de 2017. Desde el punto de vista meteorológico, el elemento más significativo fue la presencia de cizalladura horizontal del viento presente en el ámbito del aeropuerto que, debido a que éste se encontraba al máximo de frecuencia temporal de operaciones, tuvo un gran impacto en la operatividad del centro de control de área.

Los principales objetivos de este estudio son los que se muestran a continuación:

- Caracterización meteorológica del episodio de cizalladura del 10-9-2017 en LEBL.

- Análisis de la pericia de los modelos de predicción en pronosticar esta situación. 


\section{Análisis}

El análisis consiste en la evaluación crítica de lo que está ocurriendo en la atmósfera en un instante y escala espacial determinada.

En las figuras 1 y 2 , se muestra el análisis del entorno sinóptico a partir del modelo HRES-IFS del Centro Europeo de Predicción a Medio Plazo (en adelante, ECMWF) para el día 10-9-2017 a las 12 UTC.
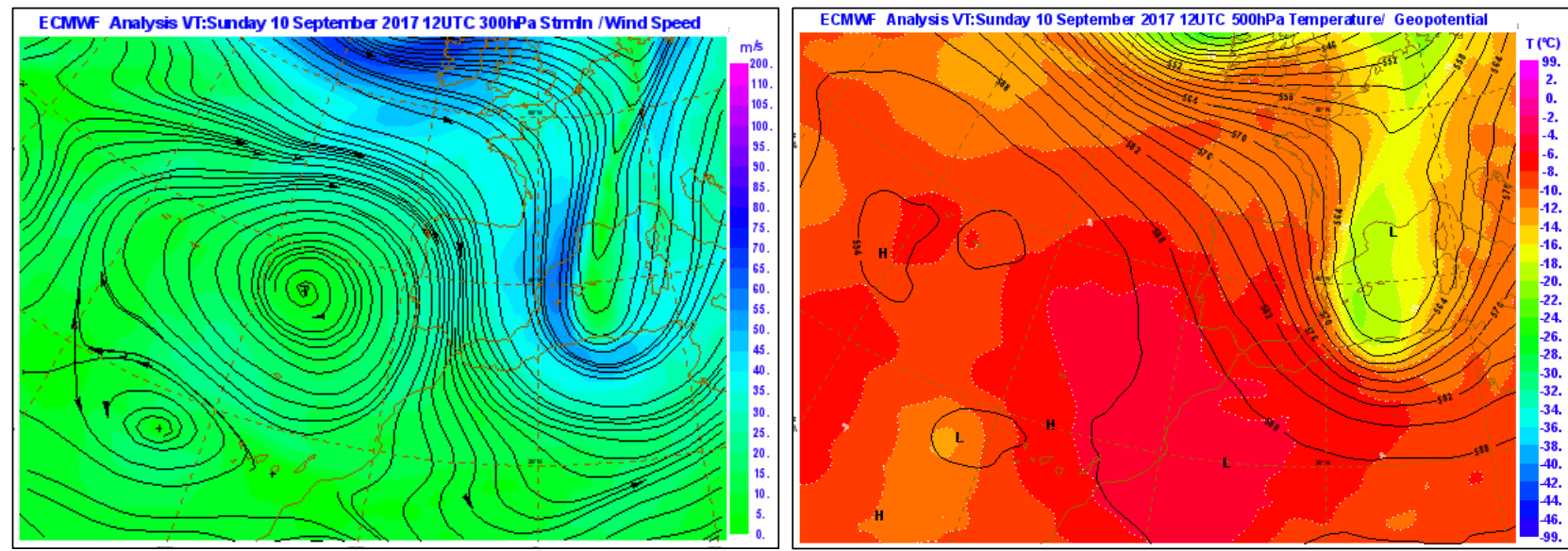

Figura 1. Análisis del modelo ECMWF para el día 10-9-2017 a las 12 UTC. A la izquierda, se representa el viento en $300 \mathrm{hPa}$. A la derecha, el geopotencial y la temperatura en $500 \mathrm{hPa}$. Fuente: Centro Europeo de Predicción a Medio Plazo (ECMWF).

En niveles medios y altos (figura 1) se observa el paso de una vaguada con un núcleo frío que alcanza los $-22{ }^{\circ} \mathrm{C}$, conducida por un máximo de viento trasero de $100 \mathrm{kt}$ y otro en su parte delantera de $80 \mathrm{kt}$, lo que posibilitó su desplazamiento progresivo hacia el este.

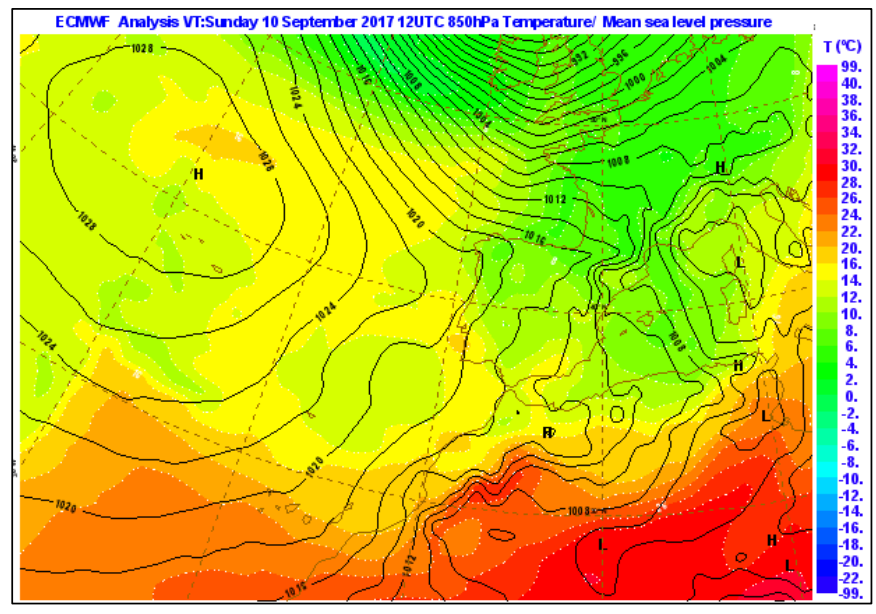

Figura 2. Análisis del modelo ECMWF para el día 10-9-2017 a las 12 UTC. Se representa la temperatura en $850 \mathrm{hPa}$ y la presión a nivel medio del mar. Fuente: Centro Europeo de Predicción a Medio Plazo (ECMWF). 
En el análisis de superficie (figura 2) se identifica un extenso anticiclón atlántico (centrado al noroeste de las islas Azores) y un centro de baja presión localizada sobre Liguria. La localización de ambos centros de acción sinópticos establece un flujo de NW sobre la Península. Éste interacciona con los obstáculos orográficos de la Península dando lugar a una configuración del campo isobárico en forma de dipolo orográfico, i.e. una mesoalta a barlovento y una mesobaja a sotavento, la cual se manifiesta por una inflexión de las isobaras en las inmediaciones de las distintas cadenas montañosas. 


\section{Diagnosis}

La diagnosis consiste en la identificación de los procesos físicos que intervienen en la formación y evolución de sistemas atmosféricos. Se trata de una labor que requiere conocimiento científico que puede lograrse gracias a un conjunto de esquemas cualitativos, ilustrativos y sintéticos denominados modelos conceptuales.

En este apartado se van a estudiar los diferentes procesos físicos involucrados en el establecimiento de un entorno favorable a cizalladura en LEBL.

El análisis sinóptico (figuras 1 y 2) muestra la afectación de la Península por un flujo del NW que algunos autores han denominado como advección septentrional ya que lo que se «advecta» es una masa de aire marítima y de origen polar que, tras interaccionar con las cordilleras alpina y pirenaica, es precursora de los siguientes sistemas de vientos regionales ageostróficos:

- En el valle del Ródano, el Mistral, que desemboca en el golfo de León.

- Entre el macizo central francés y el Pirineo, la Tramontana, que también desemboca en el golfo de León.

- Entre el Pirineo y la Ibérica, el Cierzo o Mestral, que desemboca en el mar Balear.

Los dos últimos configuran, en el nordeste de la Península, el sistema de vientos Tramontana-Mestral, muy habitual en esta configuración sinóptica. Hay que señalar que se les considera «sistema» debido a que son consecuencia de un mismo hecho sinóptico y que habitualmente soplan simultáneamente.

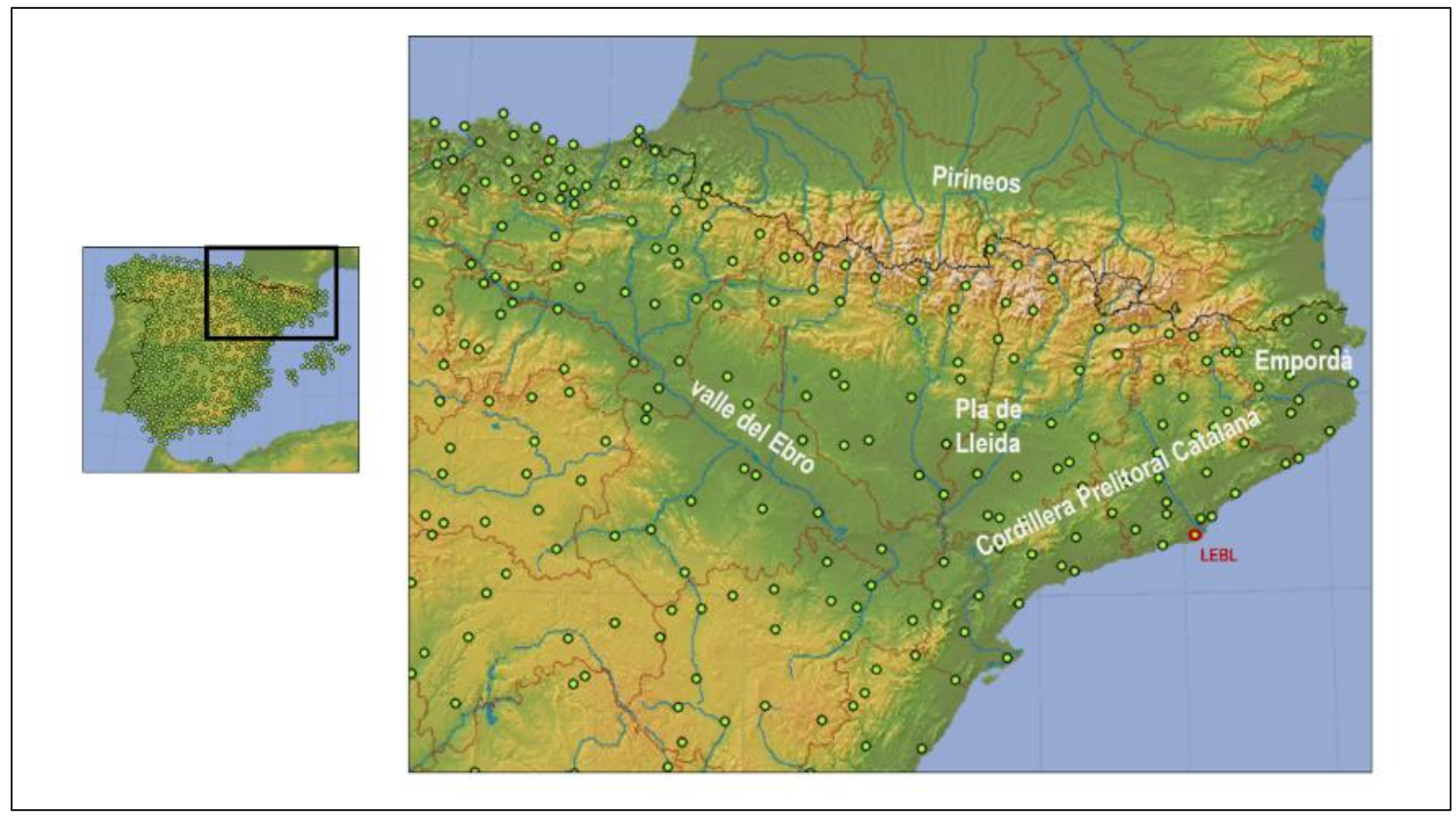

Figura 3. Área geográfica de estudio en el que se muestra la localización de las estaciones automáticas de AEMET, señalando en rojo LEBL. 
Una adecuada diagnosis requiere un afinado conocimiento de la geografía del área objeto de estudio (figura 3). Ésta se enmarca en el extremo nordeste de la Península, en la que destacan los siguientes accidentes geográficos: Pirineos, valle del Ebro, Pla de Lleida y la Cordillera Prelitoral Catalana que se extiende casi paralela al litoral hasta el Empordà.

\subsection{Caracterización mesoescalar}

La diagnosis mesoescalar es un procedimiento técnico cuyo principal objetivo es la caracterización del entorno mesoescalar de una región espacial concreta y en un instante determinado. El carácter «subjetivo» viene dado por el número de modelos conceptuales que conoce el meteorólogo, fruto de su formación y experiencia.

En este caso, la diagnosis consistirá en dar valor añadido al campo de viento en superficie, registrado en un conjunto de puntos discretos que se corresponden con las estaciones automáticas de la Agencia Estatal de Meteorología (en adelante, AEMET), mediante la identificación de los flujos que se establecen como consecuencia de la deformación del campo isobárico. Todo ello se muestra en las figuras 4 y 5, para el día 10-9-2017 a las 13 UTC, 16 UTC y 17 UTC, respectivamente.

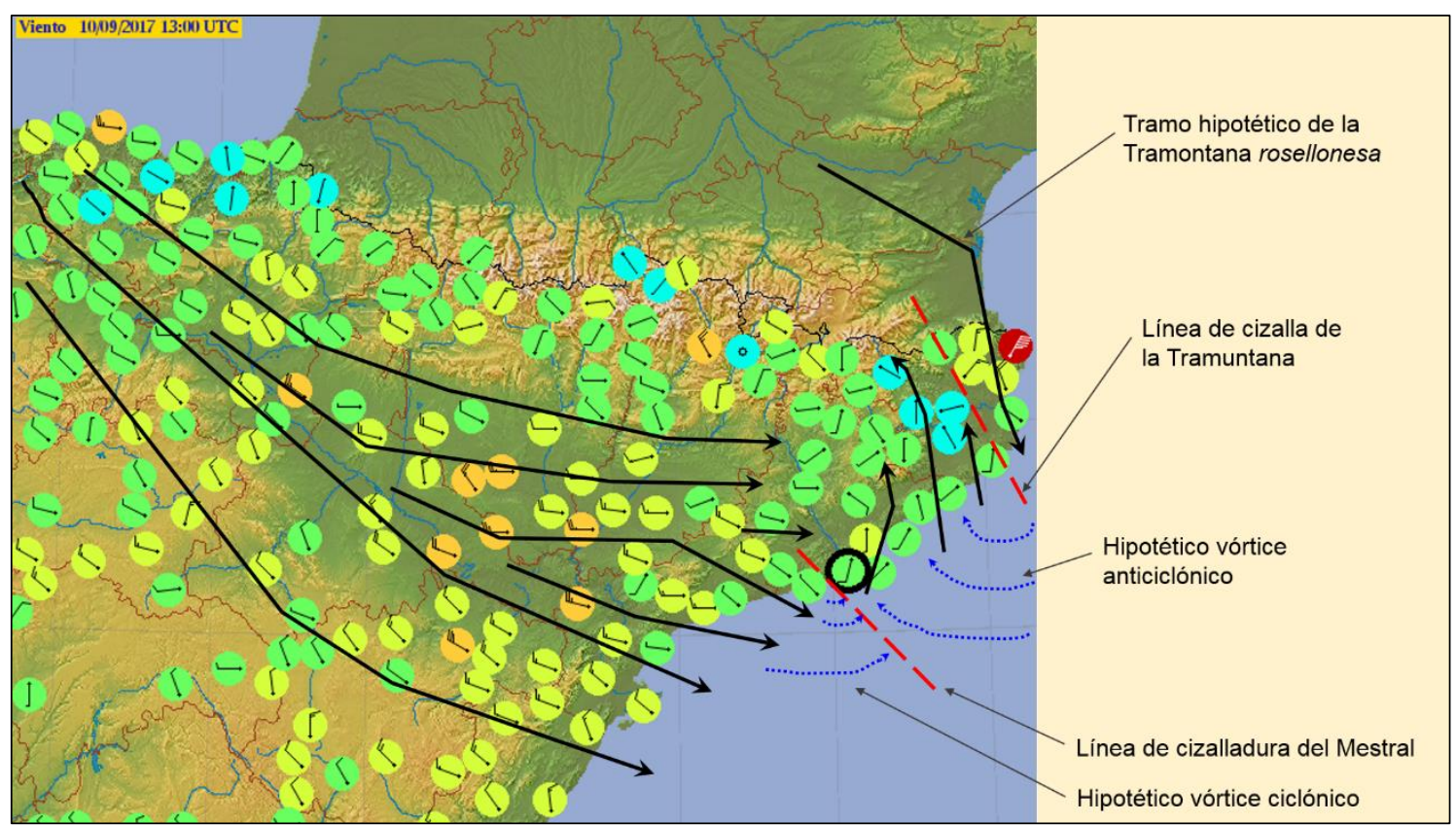

Figura 4. Diagnosis mesoescalar del día 10-9-2017 a las 13 UTC elaborado a partir de los datos de viento en superficie proporcionados por las estaciones automáticas. La gama de colores de las barbas denota la intensidad del viento.

En particular, de la figura 4 se destaca:

- Viento de componente W-NW soplando sobre el valle del Ebro, afectando a la mitad sur de Cataluña, incluyendo el Pla de Lleida, toda la provincia de Tarragona y el interior y extremo sur de la de Barcelona.

- Viento de $\mathbf{N}$ en el Empordà, que es la aceleración que sufre la Tramontana por producirse en el extremo de la cordillera pirenaica. 
- Viento de S en el litoral de Barcelona y parte de Girona. Este flujo es habitual cuando sopla el Mestral-Tramontana, el cual se genera por la curvatura anticiclónica de la Tramontana y/o curvatura ciclónica del Mestral. Ambos vórtices se producen mar a dentro. Nótese que, dada la época del año, el calentamiento diferencial mar- tierra favorecería el establecimiento de las brisas que, en el caso de la costa de Barcelona, podrían acoplarse con la circulación de dichos vórtices.
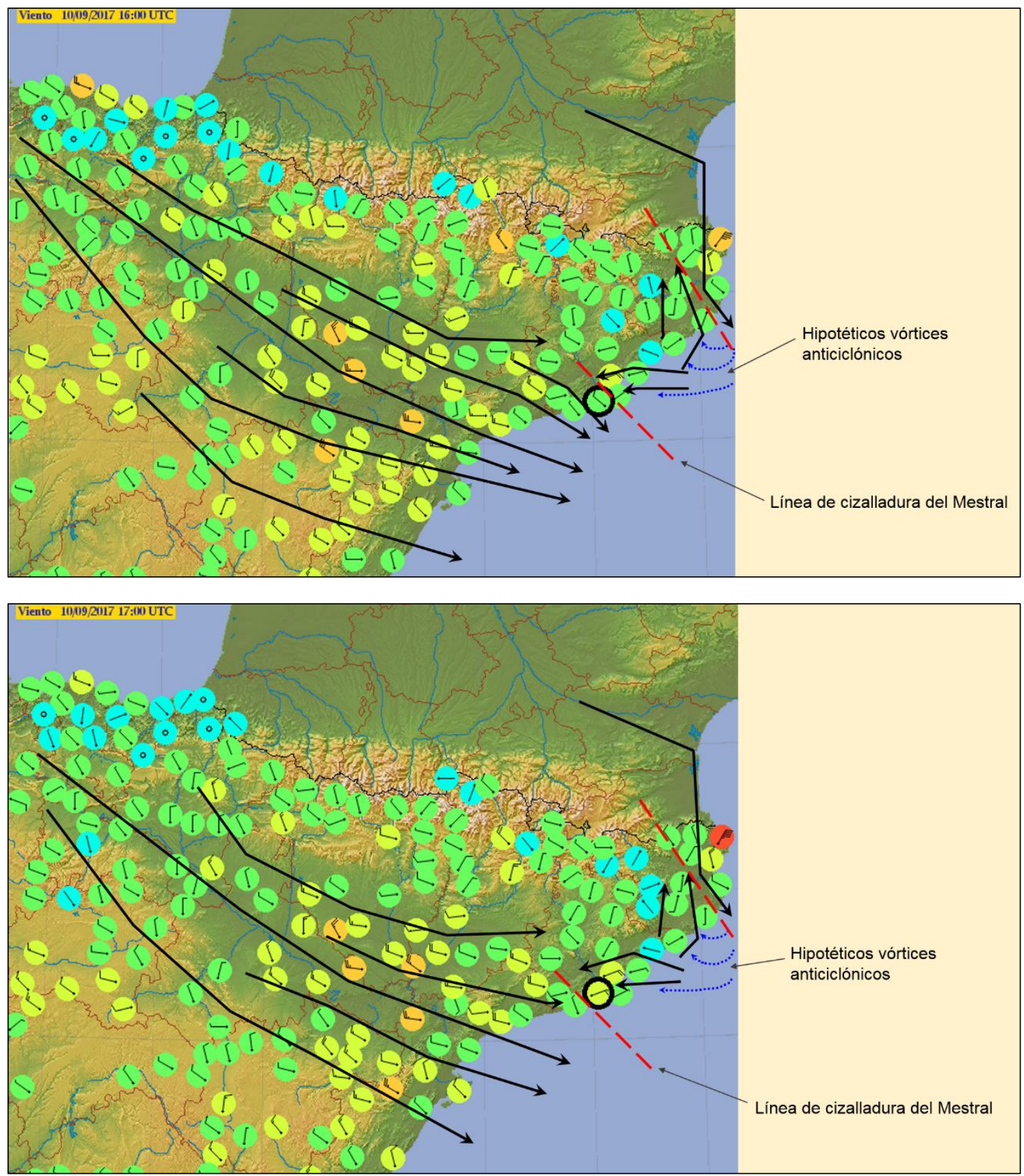

Figura 5. Diagnosis mesoescalar del día 10-9-2017 a las 16 UTC y 17 UTC elaborado a partir de los datos de viento en superficie proporcionados por las estaciones automáticas. La gama de colores denota la intensidad del viento. 
La diagnosis de las horas posteriores revela que la línea de cizalladura del Mestral cambia de posición, habida cuenta de los vientos registrados en LEBL: viento del NW a las 16 UTC (no es un «terral» en sentido «catabático seco», sino el flujo sinóptico del NW influenciado por los obstáculos orográficos) y viento del NE a las 17 UTC.

Por lo general, la situación típica de Cierzo-Mestral (viento del NW) suele afectar exclusivamente al sur de Tarragona pero en algunas situaciones, como la que se está estudiando, también lo hace a lugares situados más al norte.

\subsection{Caracterización del entorno micrometeorológico}

La disponibilidad de los datos UGDD (Unidad de Grabación de Datos Digital) supone una valiosa fuente de información ya que éstos permiten conocer el campo de viento observado en superficie cada diez minutos, en cada uno de los sensores localizados en las cabeceras de LEBL.

De esta forma, LEBL queda detallado por seis puntos (un sensor por cabecera) a diferencia del apartado anterior (figuras 4 y 5), en el que tan sólo se disponía de una sola fuente de observación (una estación automática en la cabecera de pista 25R). Este aumento de resolución espacio-temporal permite un seguimiento muy preciso de la situación e incluso, en última instancia, inferir el entorno micrometeorológico.

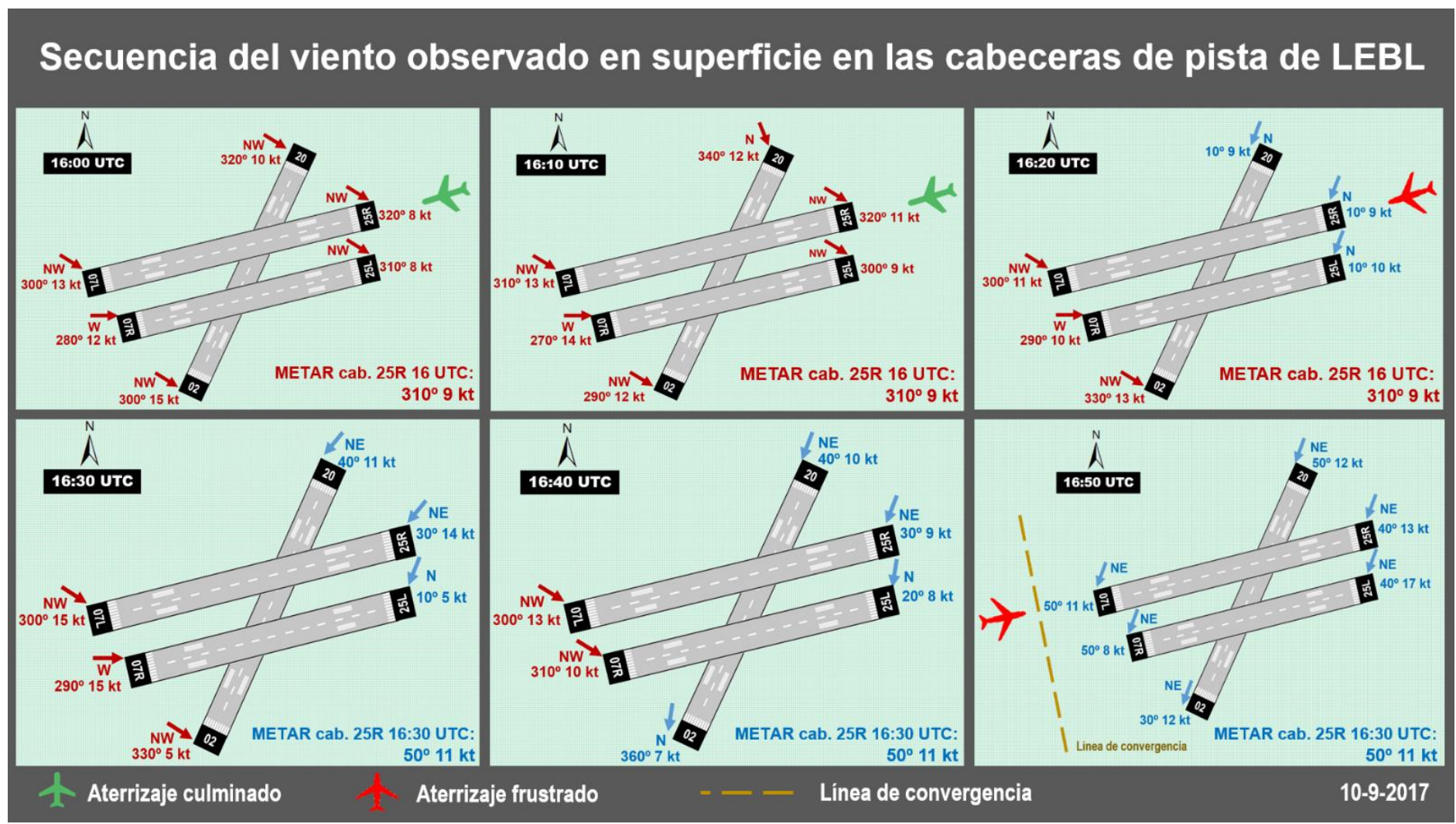

Figura 6. De izquierda a derecha y de arriba hacia abajo, secuencia de viento observado en superficie en cada una de las cabeceras de las pistas de LEBL el 10-9-2017 a las horas especificadas: desde las 16:00 hasta las 16:50, en intervalos de 10 minutos. Los colores diferencian las direcciones del viento observado en cada una de las cabeceras y las aeronaves que logran aterrizar (verde) y que frustran (rojo). Se incluye el viento cifrado en el METAR elaborado a partir de la cabecera $25 R$. 
En la figura 6 se presenta la secuencia temporal, en intervalos de diez minutos, del viento en superficie observado en cada una de las cabeceras de LEBL el día 10-9-2017. En un primer instante (16:10 UTC), aparecía un campo de viento uniforme del NW que se prolongó durante diez minutos. A lo largo de ese intervalo, la configuración del aeródromo era tal que las aeronaves aterrizaban satisfactoriamente por la cabecera $25 \mathrm{R}$, ya que, para culminar esta operación, se requiere un viento en contra o un máximo de viento a favor de $9 \mathrm{kt}$. A las 16:20 UTC, se mide por primera vez viento del $\mathrm{N}$ sobre las cabeceras de arribada. Este hecho ocasionó 5 aterrizajes frustrados (en adelante, frustradas) que fueron registradas por la torre de control (TWR) a las 16:25 UTC, lo que supuso un cambio de configuración de pista, llegando incluso a utilizar la pista transversal -con la consiguiente reducción a la mitad de la capacidad-.

En la figura se ha incluido también el viento cifrado en el METAR que estuviera en vigor, información que se elabora a partir de los datos de la cabecera 25R. Hay que tener en cuenta que el viento del METAR es un promedio en 10 minutos mientras que los representados en las cabeceras y obtenidos de la UGDD son promedios en 2 minutos.

Es interesante destacar que, a pesar de registrarse un campo de viento uniforme del NE en todas las cabeceras de pista a las 16:50 UTC, las aeronaves no podían aterrizar por la cabecera 07L. De hecho, a las 17:30 UTC, TWR registró 3 frustradas.

La caracterización mesoescalar y micrometeorológica precedentes permiten diagnosticar lo que ocurrió: entre las 16:10 UTC y las 16:50 UTC, una línea de convergencia -denotada por el cambio del viento horizontal de NW a NE- barrió paulatinamente las pistas de LEBL hasta detenerse en las cercanías de éste. Este emplazamiento imposibilitó a las aeronaves aterrizar por la 07L, a pesar de que los sensores de viento de todas las cabeceras señalaban un viento en superficie del NE, ya que sufrieron una componente de viento en cola demasiado elevada en el último tramo de la aproximación.

Así, en situaciones en las que un fenómeno de escala espacial y temporal pequeña afecta al aeropuerto, como por ejemplo la cizalladura, la información METAR no es suficiente para caracterizar la situación y, por consiguiente, deben considerarse los datos de observación proporcionados por todas las cabeceras. Además, si la línea de convergencia permanece muy próxima a la cabecera por la que se aterriza, como ocurrió en este caso, el viento registrado en dicha cabecera tampoco será representativo del viento que sufrirán las aeronaves en su aproximación.

\subsection{Modelo conceptual}

El conjunto de procesos físicos precursores del entorno de cizalladura que se está estudiando en el presente trabajo quedan sintetizados con el modelo conceptual de corriente de densidad atrapada en la costa mediterránea noroccidental descrito por González et al., (2018).

Un análisis pormenorizado de cada uno de los términos constituyentes que conforman la denominación anterior ayudará a arrojar luz sobre su significado físico: 
- Corriente: es el flujo o incursión de una masa de aire que se produce como consecuencia de una perturbación en el campo de la temperatura potencial. Es un concepto que alude a la naturaleza «dinámica» del proceso.

- Densidad: el mecanismo que lo gobierna es la diferencia horizontal de temperatura potencial entre dos masas de aire ( $y$ por ende, de densidad). Es un concepto que sugiere la naturaleza «termodinámica» del proceso.

- Atrapada en la costa: el forzamiento geográfico juega un papel esencial. La cordillera costero-catalana en el litoral de Barcelona y el efecto de Coriolis (desvío del flujo hacia la derecha en el sentido del movimiento) favorecen el confinamiento de la masa de aire más fría.

Así, lo que en un principio era una única masa de aire sinóptica y húmeda, formada en el Atlántico Norte, se convierte en dos al cruzar los Pirineos. Las condiciones orográficas a ambos lados de la cordillera se vuelven decisivas en la formación de dos masas de aire mesoescalares, de características termodinámicas contrapuestas, en la cuenca mediterránea noroccidental.

La incursión del Cierzo (o Mestral) (cálido y seco) y de la Tramontana (húmeda y fría) en el Mediterráneo estimula la formación de una línea de convergencia que progresivamente avanza hacia el lado cálido debido a la diferencia de densidad. A lo largo de ese proceso de barrido, la masa más cálida y seca se sitúa por encima de la más húmeda y fría, dando lugar a un cambio súbito en la dirección e intensidad del viento, que rola del NW-SW al NE, lo que supone un entorno favorable a cizalladura horizontal y vertical donde se localiza LEBL (figuras 7 y 8 ).

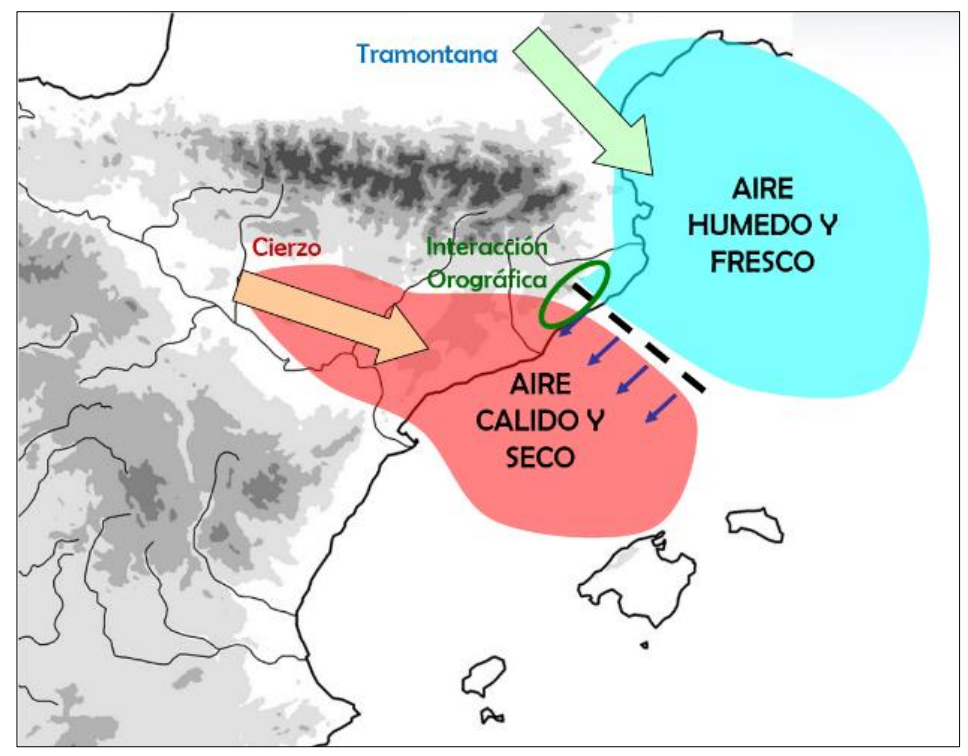

Figura 7. Esquema conceptual de una corriente de densidad atrapada en la costa de Barcelona. Los colores identifican la naturaleza térmica de las masas de aire involucradas. El avance de la masa de aire húmeda y fría sobre la más seca y cálida es precursora de un entorno favorable a cizalladura horizontal sobre el litoral de Barcelona. Fuente: González et al., 2018. 
La figura 8 es una representación idealizada que muestra la variación vertical del viento en las inmediaciones de LEBL cuando una masa de aire más húmeda y fría inyectada por un viento del NE desplaza a otra más cálida y seca que está conducida por un viento del NW. Los colores mostrados diferencian ambas masas de aire.

Otra cosa reseñable es el impacto del viento en la vertical durante la operación de aterrizaje de las aeronaves cuando se aproximan a LEBL. Así, en primer lugar, antes de las 16 UTC (figura 8, parte superior), éstas pueden alcanzar la cabecera de arribada $25 R$, mientras que en las horas posteriores a las 17 UTC (figura 8, parte inferior), las aeronaves frustran. Durante esas horas, aunque se observa un viento del NE sobre las pistas, las aeronaves en aproximación sienten, por encima de unos $1000 \mathrm{ft}$ o a veces menos, viento de cola que les afecta a su sustentación. Por este motivo, cuando alcanzan la altura de decisión, se ven obligados a volver al aire y recorrer un circuito de espera hasta efectuar un nuevo intento de toma de tierra por la cabecera 07L.

La configuración del LEBL se establece fundamentalmente según el viento observado en las pistas. En la figura 8 se observa cómo se pasó de la configuración $\mathrm{W}$ inicial (aterrizaje por la cabecera 25R y despegue por la $25 \mathrm{~L}$ hacia el oeste) a la E (aterrizaje por la cabecera 07L y despegue por la 07R hacia el este) el día 10-9-2017. A pesar de este cambio táctico, las aeronaves no pudieron culminar su aproximación, debido a que a la línea de convergencia se situó en las inmediaciones de LEBL, estableciendo un viento cruzado y con una componente en cola en la mayor parte de la senda de planeo.

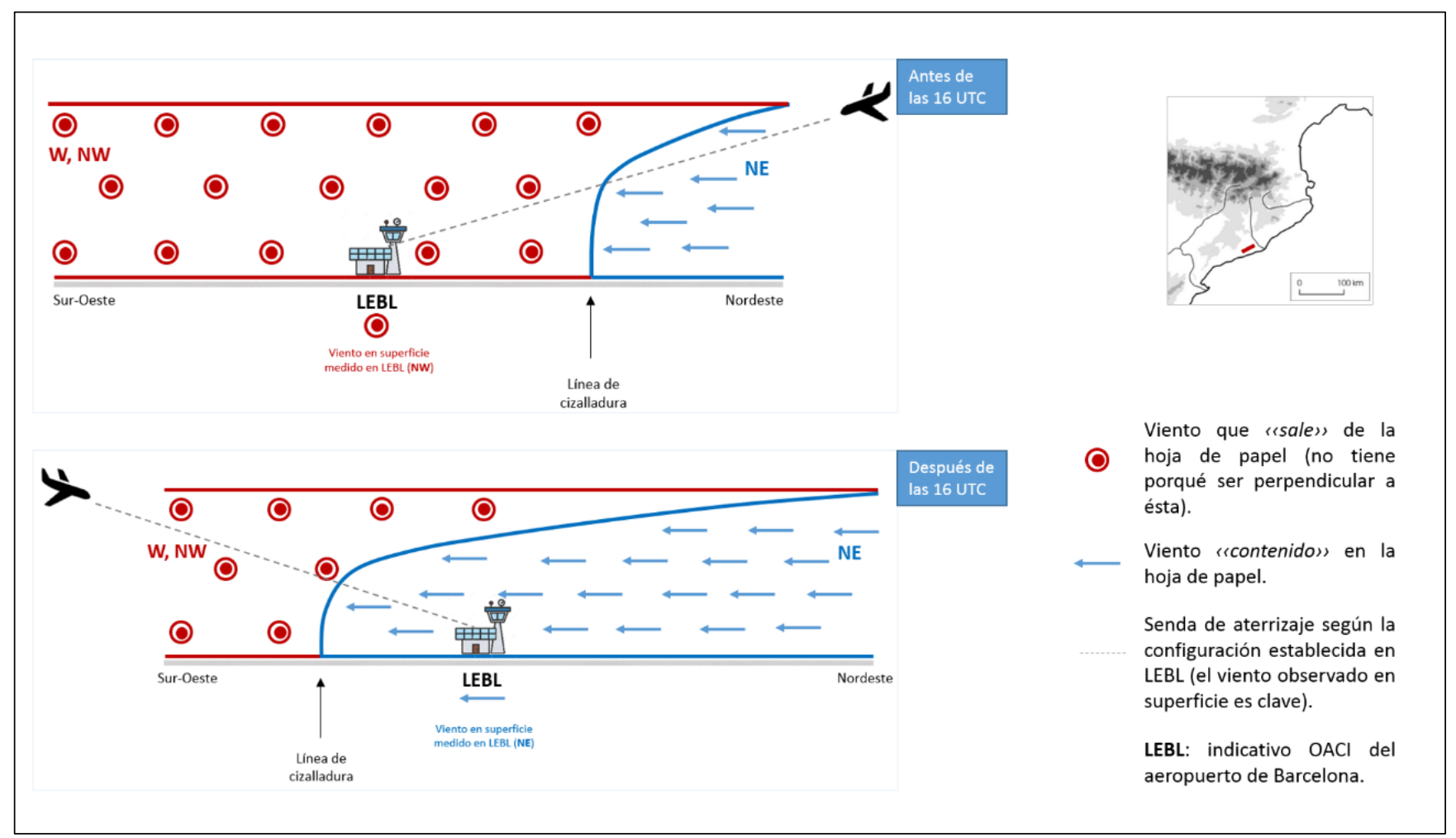

Figura 8. Imagen conceptual de la variación del viento en las inmediaciones de LEBL según una sección vertical sobre el eje de la pista 25R-07L y su impacto en las operaciones de aproximación y aterrizaje. Los colores diferencian los dos tipos de viento observados. 
Los ingredientes fundamentales para la ocurrencia de este fenómeno mesoescalar son los siguientes:

- Gradiente horizontal de densidad: La frontera entre dos masas de aire de distintas densidades inicia la corriente de densidad que se desplaza hacia el SW de forma cuasi paralela a la cordillera costero-catalana.

- Efecto de Coriolis: favorece que la masa de aire húmeda y fría, según avanza hacia el SW, se desvíe progresivamente hacia la derecha, es decir, «tierra adentro».

- Entorno atmosférico estable: supone un impedimento para que la masa de aire húmeda y fría remonte la adyacente Cordillera Prelitoral Catalana.

- Curvatura del litoral de Barcelona: podría ayudar a que la corriente de densidad disminuya su velocidad y finalmente quede retenida en las cercanías de LEBL.

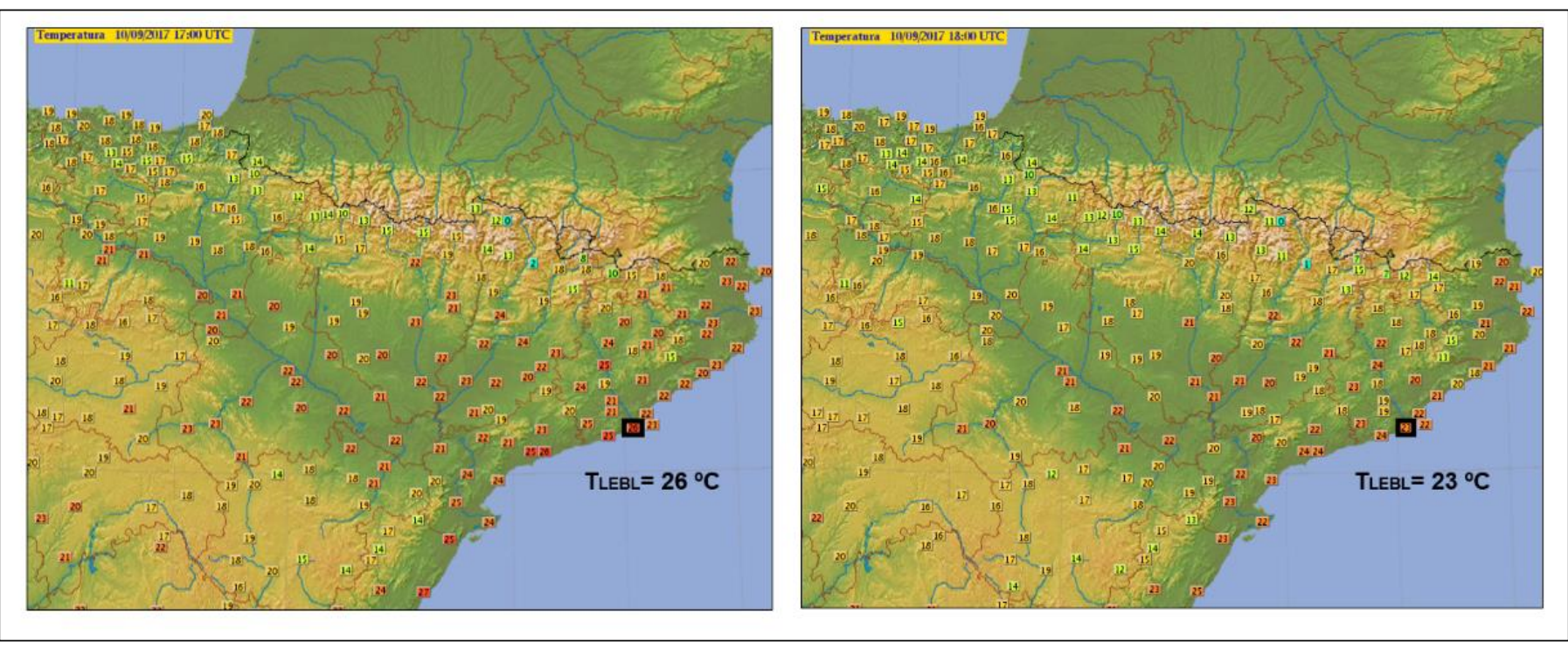

Figura 9. Temperatura observada en superficie por las estaciones automáticas el 10-9-2017 a las 17 UTC (izquierda) y a las 18 UTC (derecha).

En la figura 9 se aprecia la evolución entre las 17 UTC y 18 UTC de la temperatura observada en superficie. Los datos procedentes de las estaciones automáticas de LEBL y la más inmediata (Sitges) confirman cómo la masa de aire más fría (más densa) barrió progresivamente el litoral barcelonés hasta detenerse entre las citadas estaciones (tabla I).

Hay que advertir que la identificación de una masa de aire no puede hacerse únicamente en base a su temperatura en superficie. Lo más lógico sería combinarla con la humedad relativa en el concepto de temperatura pseudoequivalente, de gran interés debido a su carácter conservativo en procesos de evaporación y condensación e interacción con la orografía. Una forma objetiva de diagnosticar la posición de línea de convergencia sería mediante el gradiente horizontal de esta magnitud. 


\begin{tabular}{|c|c|c|c|c|c|c|c|c|c|c|c|c|}
\hline & \multicolumn{4}{|c|}{ Viento (grados, kt) } & \multicolumn{4}{|c|}{ Temperatura $\left({ }^{\circ} \mathrm{C}\right)$} & \multicolumn{4}{|c|}{ Humedad relativa (\%) } \\
\hline & \multicolumn{4}{|c|}{ Horas (UTC) } & \multicolumn{4}{|c|}{ Horas (UTC) } & \multicolumn{4}{|c|}{ Horas (UTC) } \\
\hline & 13 & 16 & 17 & 18 & 13 & 16 & 17 & 18 & 13 & 16 & 17 & 18 \\
\hline $\begin{array}{l}\text { Aeropuerto } \\
\text { Barcelona- El } \\
\text { Prat }(0076)\end{array}$ & $\begin{array}{l}200^{\circ} \\
9 \mathrm{kt} \\
\mathrm{SW}\end{array}$ & $\begin{array}{l}310^{\circ} \\
10 \mathrm{kt} \\
\mathrm{NW}\end{array}$ & $\begin{array}{c}70^{\circ} \\
17 \mathrm{kt} \\
\mathrm{NE}\end{array}$ & $\begin{array}{c}60^{\circ} \\
11 \mathrm{kt} \\
\mathrm{NE}\end{array}$ & 24.8 & 28.8 & 25.8 & 23 & 40 & 21 & 45 & 54 \\
\hline $\begin{array}{l}\text { Sitges- } \\
\text { Vallcarca } \\
(0073 X)\end{array}$ & $\begin{array}{l}320^{\circ} \\
3 \mathrm{kt} \\
\mathrm{NW}\end{array}$ & $\begin{array}{l}320^{\circ} \\
4 \mathrm{kt} \\
\mathrm{NW}\end{array}$ & $\begin{array}{l}340^{\circ} \\
4 \mathrm{kt} \\
\mathrm{NW}\end{array}$ & $\begin{array}{l}340^{\circ} \\
3 \mathrm{kt} \\
\mathrm{NW}\end{array}$ & 26.4 & 27 & 25.4 & 24 & 27 & 30 & 34 & 38 \\
\hline
\end{tabular}

Tabla I. Análisis de la tendencia local del viento, temperatura y humedad relativa en superficie medidas por las estaciones automáticas de LEBL y Sitges.

La formación y posterior evolución de este fenómeno mesoescalar puede resumirse en el cuadro mostrado en la figura 10.

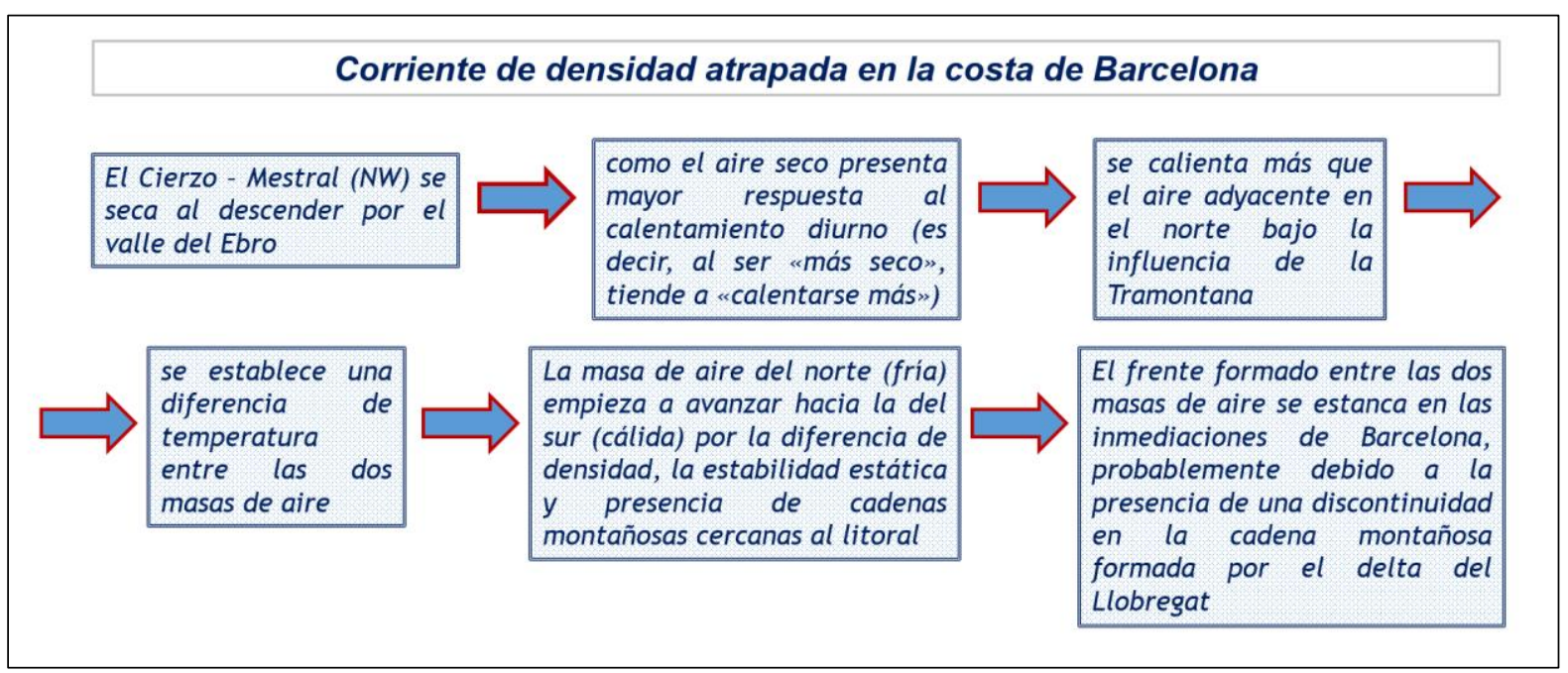

Figura 10. Formación y posterior evolución de la corriente de densidad mesoescalar atrapada en la costa de Barcelona. 


\section{Predicción}

La predicción consiste en la estimación del estado futuro de la atmósfera en un rango espacial y temporal determinado. Se trata de un proceso secuencial, jerarquizado, que requiere conocimiento científico y además, de difícil solución, habida cuenta de la naturaleza caótica de la atmósfera.

La predicción, al igual que el análisis y el diagnóstico, no puede resolverse de manera global. Es necesaria la separación de las escalas espacio- temporales involucradas. Para ello, es fundamental partir de un marco sinóptico (identificando los principales centros de acción), pasando posteriormente al ámbito de la mesoescala (detallando los vientos locales) hasta finalmente llegar a la microescala, lo que conlleva indagar en las variaciones instantáneas del viento en superficie que podrían delatar una situación favorable a cizalladura. En la figura 11 se muestra de forma gráfica y concisa lo comentado.

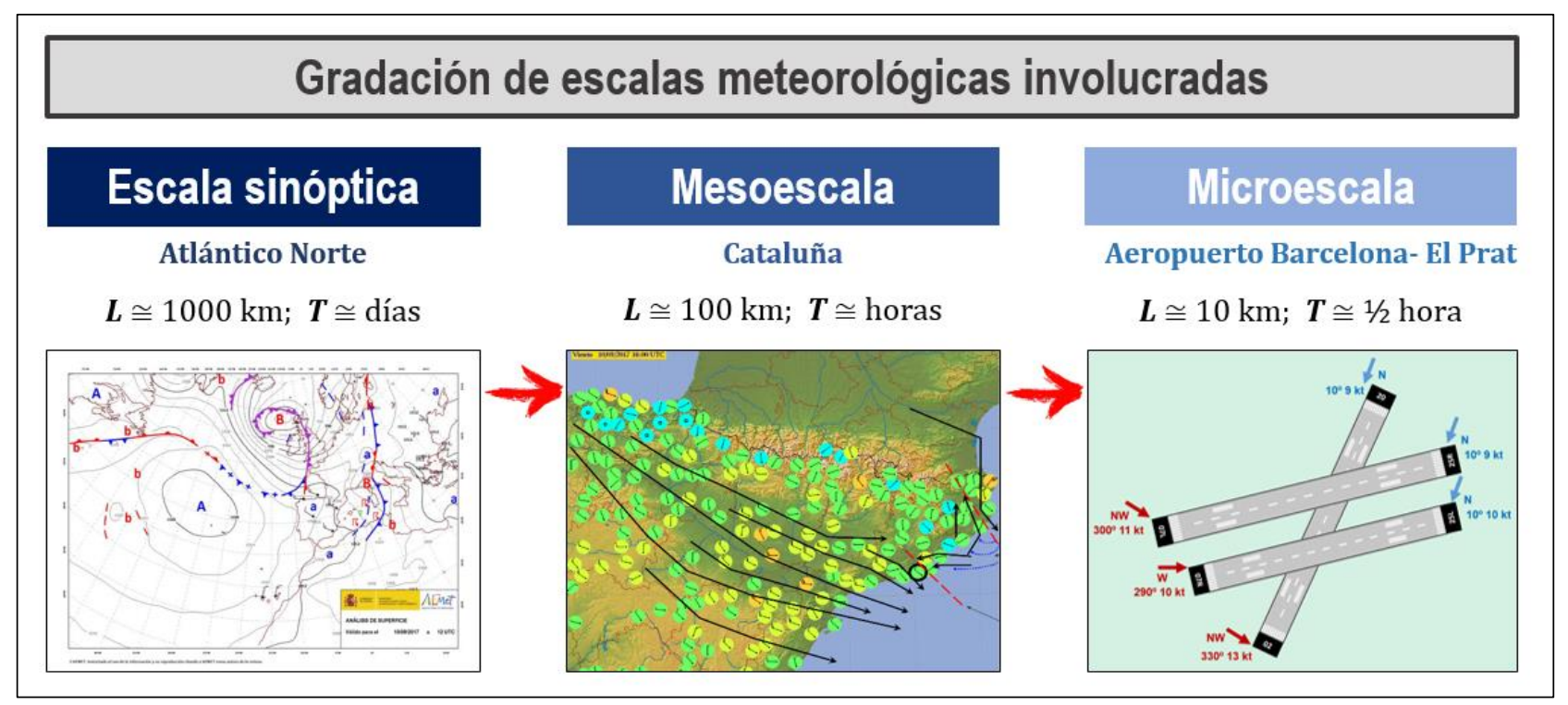

Figura 11. Relación de escalas meteorológicas involucradas en la predicción del entorno favorable a cizalladura del día 10-9-2017 en LEBL, con sus correspondientes órdenes de magnitud espaciotemporales característicos.

En esta sección se analizará la pericia de los modelos en predecir el episodio de cizalladura del 10-9-2017 en LEBL. Hay que advertir que se está tratando de pronosticar un fenómeno que se ubica en la microescala (Markowski et al., 2010). Por este motivo, a priori sería incompleta la consideración única de los campos previstos por un modelo determinista. Es necesario el uso de modelos probabilistas, ya que, la incertidumbre espacio-temporal asociada a este fenómeno «tan local», no es desdeñable.

Algunos interrogantes que subyacen de la anterior reflexión podrían ser tales como: ¿de qué herramientas dispone AEMET para la predicción de un fenómeno tan local como el que se está estudiando? ¿El estado del arte de la predicción permitiría elaborar un pronóstico determinista de este fenómeno? 


\subsection{Modelo de predicción determinista HARMONIE-AROME operativo}

El modelo HARMONIE-AROME operativo en AEMET está desarrollado por los consorcios ALADIN y HIRLAM. Se trata de un modelo de predicción determinista, de área limitada, no hidrostático (convection permiting), con un núcleo dinámico basado en una discretización semi-lagrangiana y semi-implícita, cuya resolución horizontal es de 2.5 $\mathrm{km}$, con 65 niveles en la vertical. Realiza ciclos de asimilación cada 3 horas. De esta forma, cada 3 horas se liberan predicciones cuya validez llega a $\mathrm{H}+48$ (Bengtsson et al., 2017).

En este caso, se evaluará el viento pronosticado a 10 metros para el día 10-9-2017 en la pasada de las 6 UTC.

La resolución horizontal y vertical permite «capturar» el forzamiento geográfico. Por otra parte, como se trata de un modelo determinista, elaborará un sólo pronóstico para esta variable que puede presentarse con bastante detalle en el área donde se localiza LEBL (figura 12).

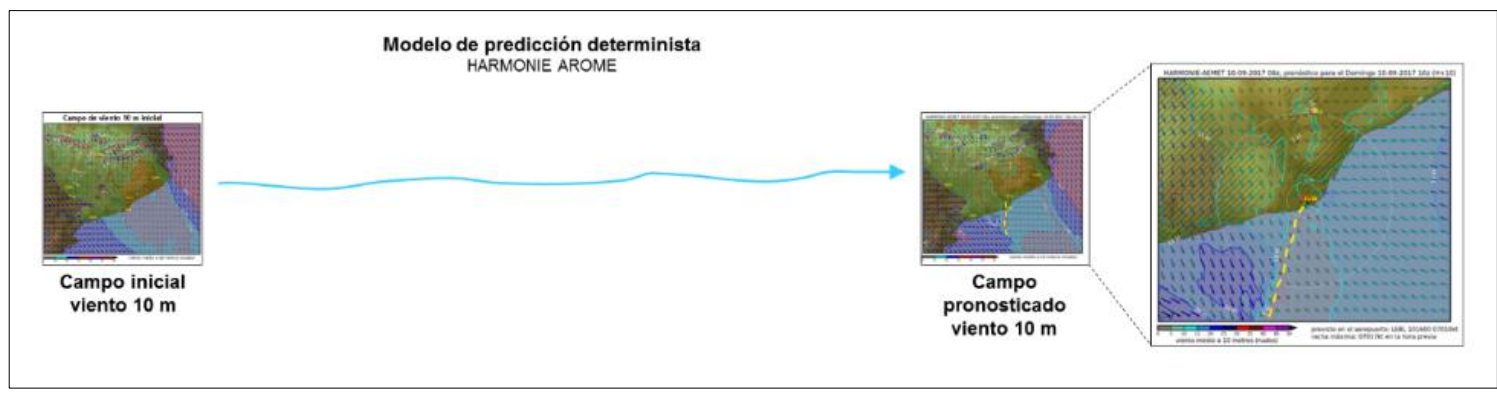

Figura 12. Modelo de predicción determinista HARMONIE-AROME, cuya resolución horizontal es de $2.5 \mathrm{~km}$. A partir de un análisis previo, se pronostica un sólo escenario.

En la figura 13 se muestra el viento pronosticado a 10 metros para el día 10-9-2017 en los alcances $\mathrm{H}+8$ (10-9-2017 a las 14 UTC) y H+11 (10-9-2017 a las 17 UTC) en la Península. Se puede apreciar notablemente el establecimiento de dos ramales bien definidos de componente N-NW asociados al sistema de vientos Cierzo, Mestral y Tramontana, citados anteriormente, apareciendo entre ambos una zona con un viento entre flojo y variable.

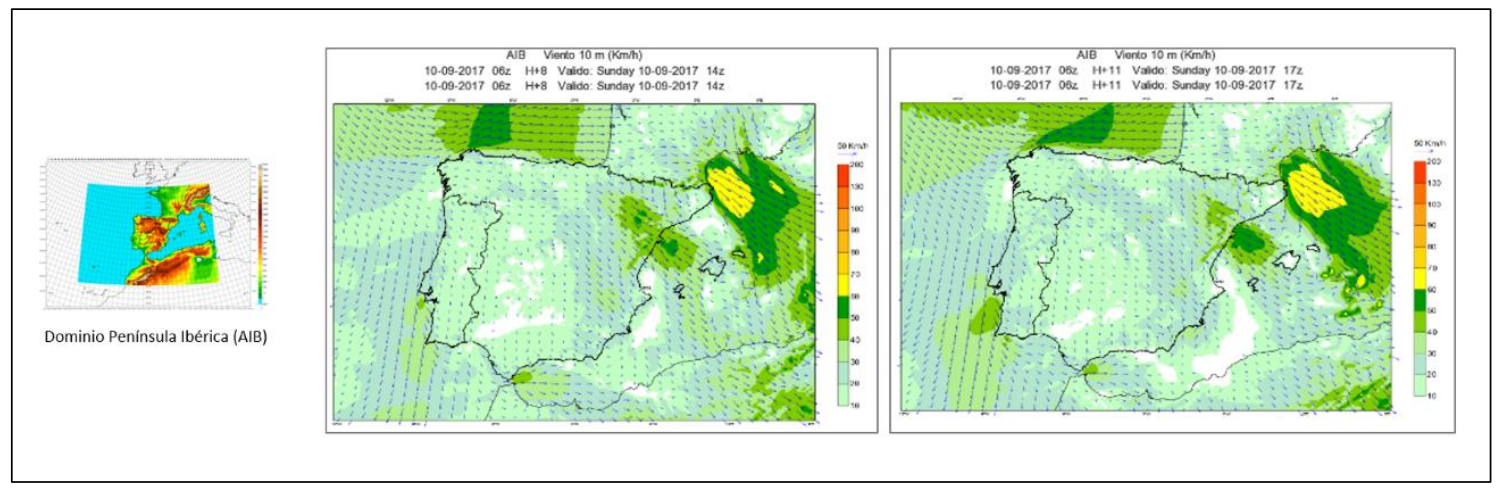

Figura 13. Viento a 10 metros pronosticado por HARMONIE-AROME en el área de la Península para el día 10-9-2017 en la pasada de las 6 UTC y alcances $\mathrm{H}+8$ y H+11. La escala de colores denota la velocidad y las flechas indican la dirección. 
En la figura 14 se presenta el viento pronosticado a 10 metros para el día 10-9-2017 a las 15:00 UTC y 16:00 UTC (alcances $\mathrm{H}+9$ y $\mathrm{H}+10$, respectivamente). Puede concluirse que el modelo sí pudo ver la línea de convergencia, habida cuenta de la discontinuidad en el viento horizontal aunque temporalmente adelantó ligeramente su paso por LEBL. Además, el modelo pronosticó un viento en las inmediaciones de LEBL más del E que del NE; este último fue el observado en el aeropuerto tras el paso de la línea de convergencia (y previsto en el TAF, véase el apartado de Predicción aeronáutica).

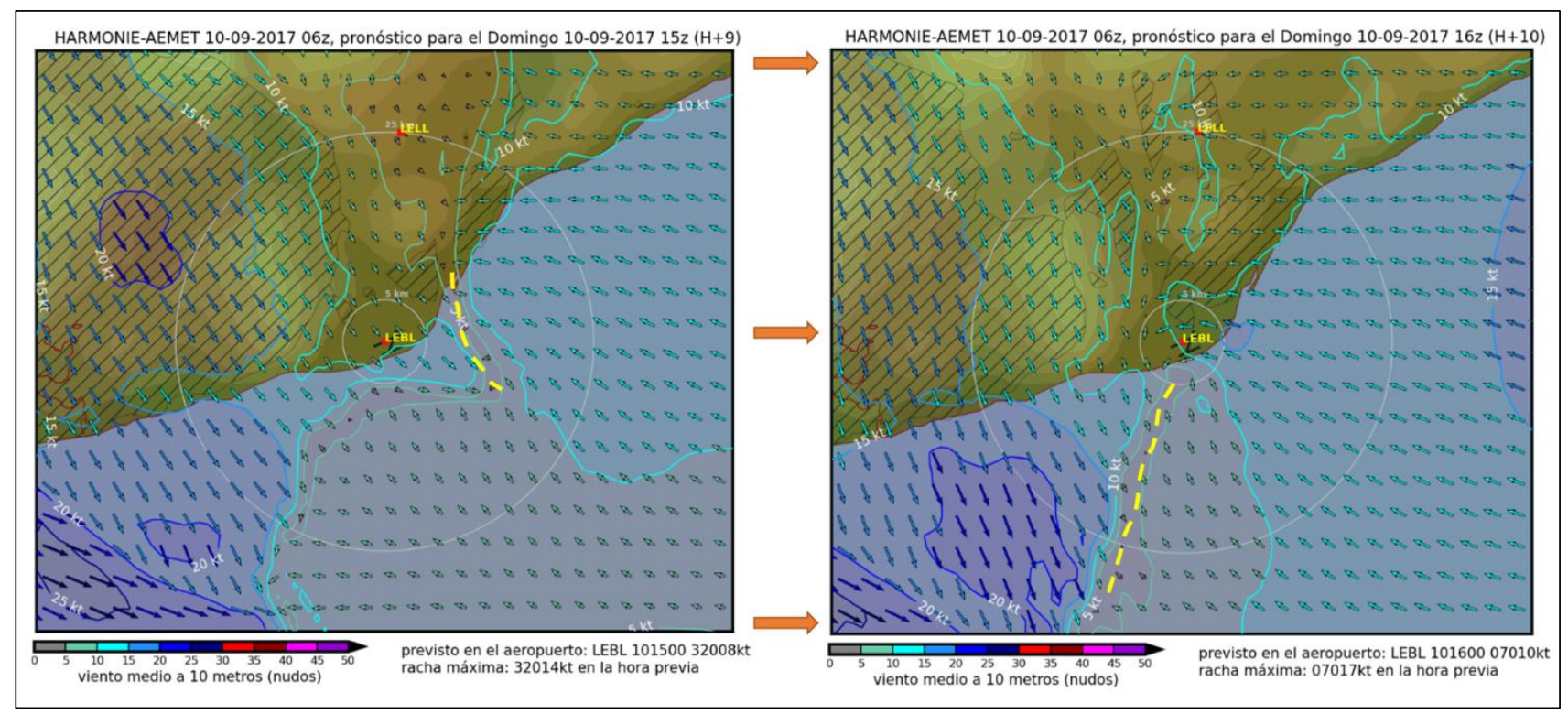

Figura 14. Viento a 10 metros pronosticado por el modelo determinista HARMONIE-AROME en la pasada de las 6 UTC y los alcances $H+9$ y $H+10$. Se incluye el trazado subjetivo de la línea de convergencia en línea discontinua y color amarillo.

Por otra parte, un meteograma es un recurso gráfico en el que se representa la evolución prevista para una determinada variable en un punto en forma de serie temporal. En la figura 15 se resume, entre otras, el pronóstico del viento a 10 metros en LEBL para los distintos alcances, según la pasada operativa de las 6 UTC del modelo HARMONIE-AROME del día 10-9-2017.

En la tercera gráfica empezando desde arriba, la línea continua azul representa la dirección del viento previsto en LEBL mientras que los puntos denotan el viento observado cifrado en el METAR. Lo más destacado es la gran dispersión que se observa en la dirección, en torno a las 16 UTC, de ahí la dificultad que tiene el modelo en pronosticar, de un modo determinista, la posición de la línea de convergencia sobre el aeropuerto. La dispersión observada es sinónimo de «incertidumbre» e indicio de «baja predecibilidad».

La forma de construir esta herramienta ilustrativa de gran interés para los predictores aeronáuticos es la siguiente: sobre la rejilla del modelo, se traza un círculo de radio igual a 10 kilómetros sobre el aeropuerto. Sobre todos los puntos de rejilla que quedan embebidos, el modelo calcula las variables de pronóstico.

Hay que señalar que, al tratarse de la predicción de un fenómeno mesoescalar cuya afectación se circunscribe a una región «muy local», sería incompleto evaluar el 
entorno previsto en el punto de rejilla más cercano a LEBL. Por este motivo hay que considerar los puntos de rejilla más cercanos.

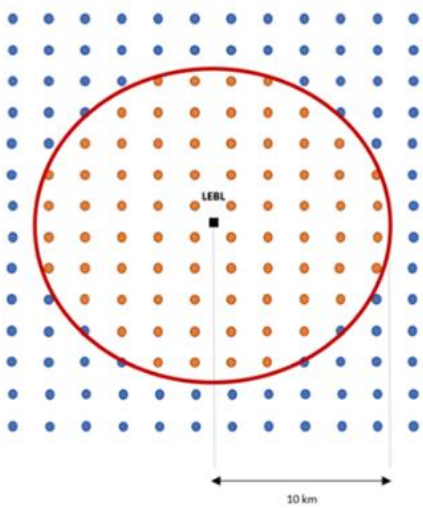

Puntos de rejilla HARMONIE AROME (resolución horizontal de $2.5 \mathrm{~km}$ )

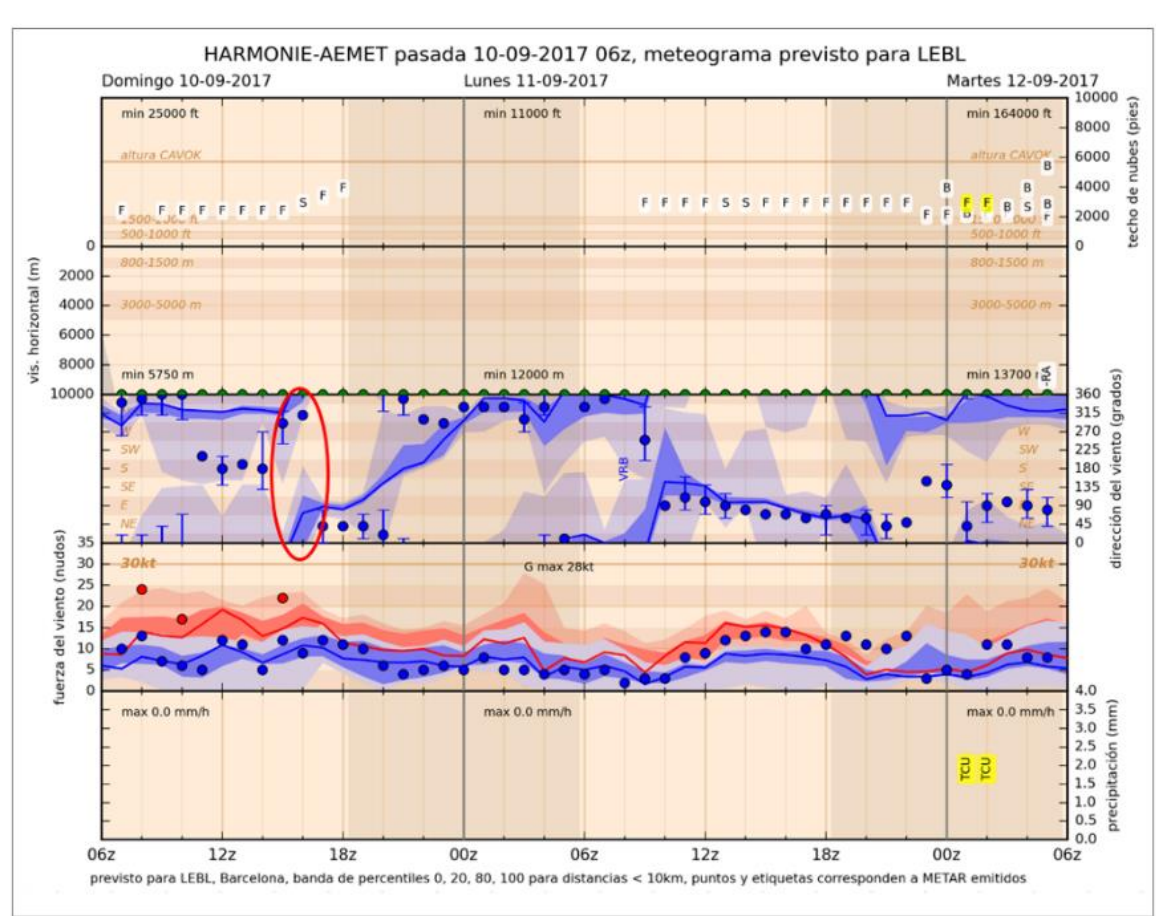

Figura 15. Meteograma calculado con el pronóstico de HARMONIE-AROME para el día 10-9-2017 en LEBL. En la parte izquierda se ilustra la forma de cálculo (explicación en el texto).

\subsection{Secciones verticales previstas por HARMONIE-AROME operativo}

Las secciones verticales de determinadas variables previstas por el modelo HARMONIEAROME operativo son de gran utilidad pues permiten, entre otras cosas, evaluar la posibilidad de presencia de cizalladura en un plano y en las proximidades a LEBL.

En la figura 16 se muestra un ejemplo de sección vertical sobre el eje de la pista. Las dos sendas de aproximación permiten dividir el entorno espacial en dos partes bien diferenciadas: las aeronaves que se aproximan por el lado derecho proceden del Maresme (ENE) mientras que las del izquierdo lo hacen desde el Garraf (WSW). 


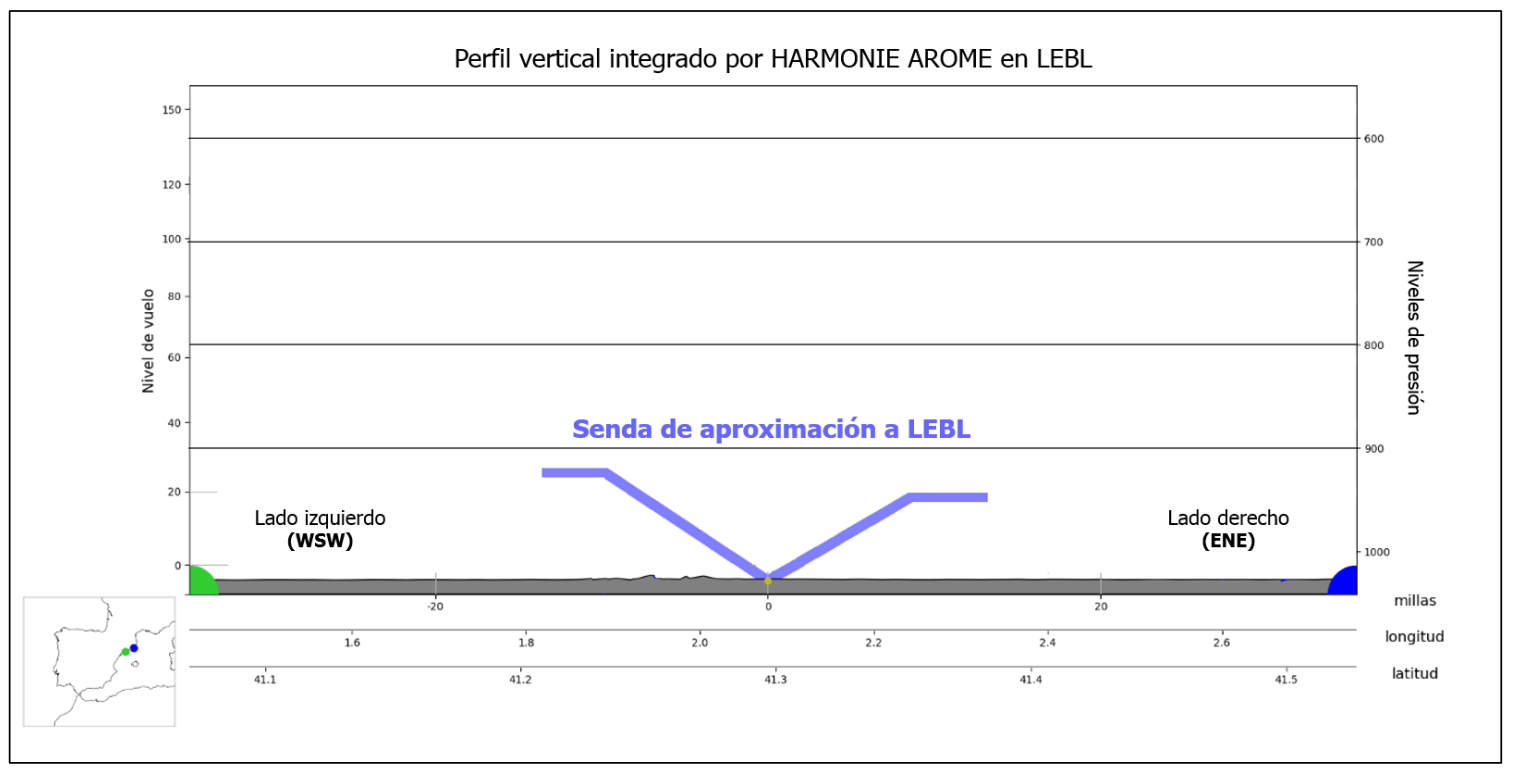

Figura 16. Sección vertical a partir del modelo HARMONIE-AROME sobre el eje de la pista $07 / 25$ en LEBL. Las sendas de aproximación a LEBL dividen el entorno espacial en dos partes (lados izquierdo y derecho).

A continuación se van a analizar los perfiles verticales de la temperatura potencial y del número de Richardson ya que ambos cuantifican la estabilidad atmosférica. En la figura 17, se representan para los alcances $\mathrm{H}+9$ (15 UTC) y $\mathrm{H}+12$ (18 UTC), respectivamente:

Interpretación según la temperatura potencial (figura 17): Una capa atmosférica es estable si la temperatura potencial aumenta con la altura e inestable si disminuye con la altura. Habrá estratificación neutra si el gradiente vertical es próximo a cero.

Al ENE del aeropuerto (parte derecha) se puede identificar una masa de aire estable (aumento vertical de la temperatura potencial) mientras que al WSW (parte izquierda) hay una masa de aire con estratificación neutra en capas bajas debido a que la temperatura potencial es prácticamente constante con la altura.

En el alcance posterior $(\mathrm{H}+12$, imagen inferior) se observa la variación de la estabilidad como consecuencia del avance de la masa de aire más fría desde el nordeste.

Interpretación según el número de Richardson (figura 17): el número de Richardson es el cociente entre la estabilidad y la cizalladura. Se trata de un índice adimensional y diagnóstico que cuantifica la posibilidad de presencia de turbulencia en un determinado entorno. De esta forma, cuanto menor sea la estabilidad y mayor la cizalladura, más pequeño será éste y, consecuentemente, mayor posibilidad de presencia de turbulencia.

En el alcance $\mathrm{H}+9$ (imagen superior) en lo que respecta al número de Richardson, la configuración es tal que, a la derecha, se observan los valores más bajos en capas próximas a la superficie, mientras que a la izquierda, éstos llegan hasta capas más altas (hasta $800 \mathrm{hPa}$ ). Así, en la parte izquierda se tiene una masa de aire más turbulenta que en la parte derecha. 
En el alcance posterior $(\mathrm{H}+12$, imagen inferior) se observa que las proximidades a $\mathrm{LEBL}$ se han estabilizado, habida cuenta de la disminución del número de Richardson en la vertical.
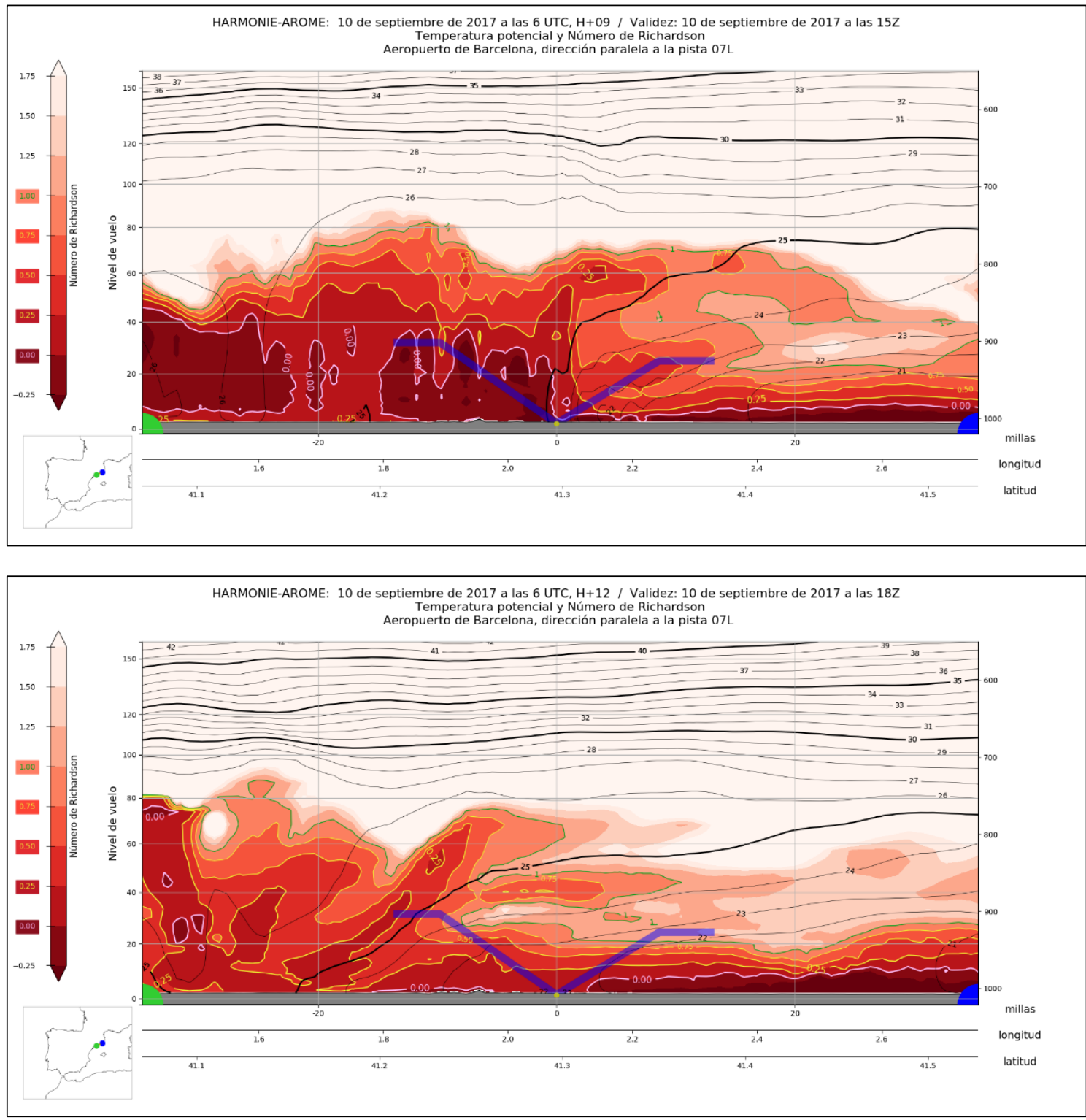

Figura 17. Perfiles verticales de temperatura potencial y número de Richardson integrados por HARMONIE-AROME. Pasada del 10-9-2017 a las 6 UTC. Alcances: H+9 (15 UTC) y H+12 (18 UTC).

Por último, es interesante analizar la variación vertical simulada por el modelo en los mismos alcances para las variables de viento horizontal (barbas) y la energía cinética turbulenta (TKE) (área en amarillo).

Interpretación según el viento horizontal (figura 18): Por debajo del nivel isobárico de $900 \mathrm{hPa}$ se observa un flujo del SE (el viento observado fue del NE) en la parte derecha y otro del NW en la parte izquierda. Así, se puede identificar la zona de convergencia entre ambas masas de aire en la zona de aproximación a la cabecera 25. 
En el alcance posterior se puede comprobar el avance del flujo del SE hacia la parte izquierda, quedando la zona de convergencia alrededor del inicio de la aproximación por la cabecera 07.

Interpretación de la energía cinética turbulenta (TKE) (figura 18): Los valores altos de TKE se corresponden con un entorno más turbulento. En este caso, aunque no se aprecian valores excesivamente altos, se comprueba el avance de la masa de aire menos turbulenta de la parte derecha hacia la parte izquierda.
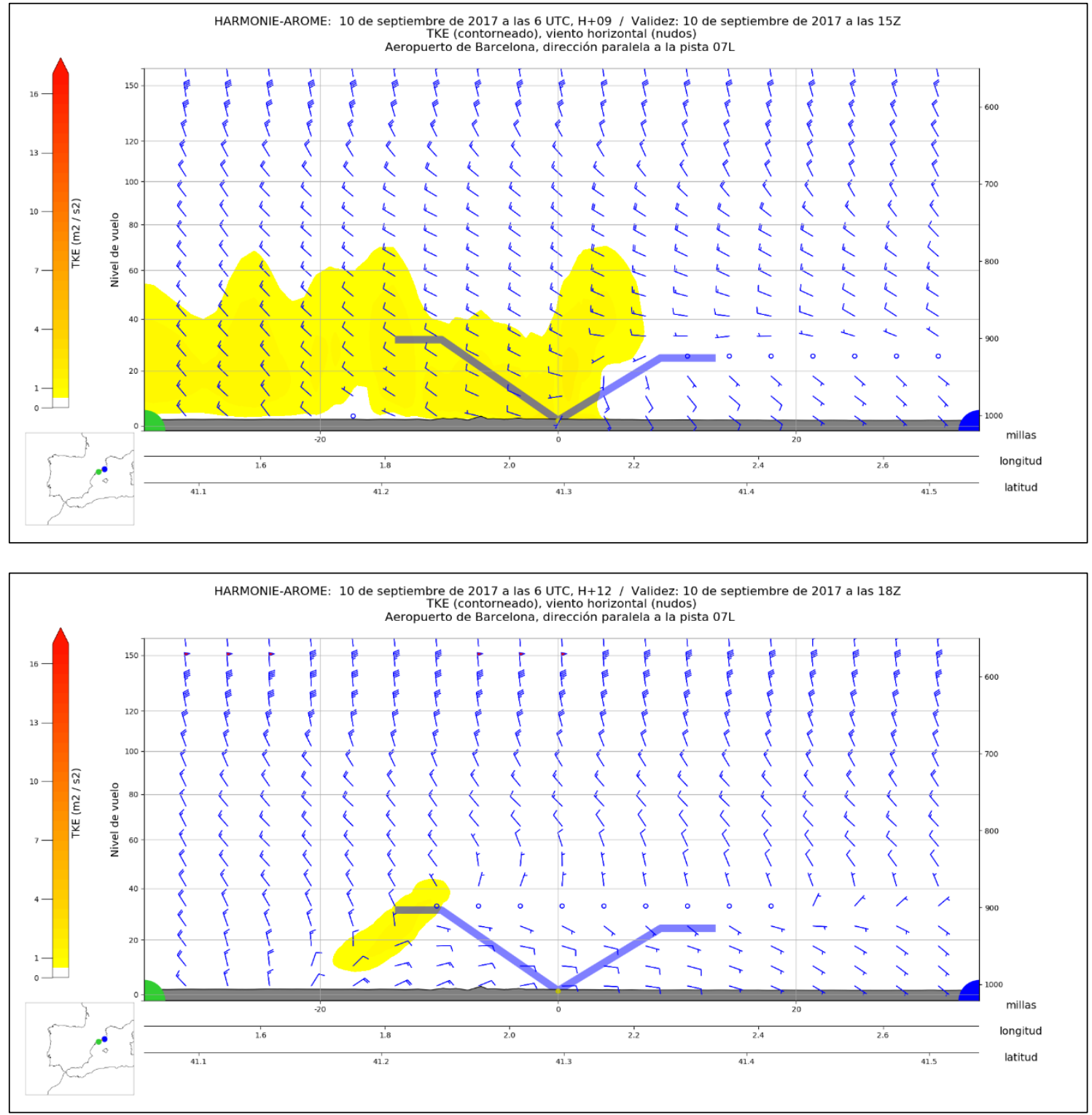

Figura 18. Perfiles verticales de viento horizontal y energía cinética turbulenta (TKE) integrados por HARMONIE-AROME. Pasada del 10-9-2017 a las 6 UTC. Alcances: H+9 (15 UTC) y H+12 (18 UTC).

Las tres conclusiones más relevantes que se derivan del análisis de las secciones verticales son las siguientes: 
- Las variables analizadas constituyen unos buenos trazadores pues permiten identificar las dos masas de aire involucradas en este episodio.

- En ambos casos, se confirma el modelo conceptual propuesto en la figura 8.

- Las secciones verticales simuladas por el modelo HARMONIE-AROME constituyen una herramienta útil en la predicción de cizalladura horizontal y vertical en la aproximación a LEBL.

\subsection{Modelo de predicción determinista HARMONIE-AROME 1 km}

Un aumento de resolución de un modelo de predicción puede ser sinónimo de mejoría en cuanto a la capacidad de simular los procesos físicos que tienen lugar en la atmósfera. Ahora bien, esta «alternativa numérica» puede generar soluciones no deseadas.

En efecto, a pesar de la utilización de esquemas de discretización numéricamente estables que permiten aumentar la resolución horizontal, pueden aparecer inestabilidades relacionadas con la acumulación artificial de energía en las escalas más pequeñas del modelo, comportamiento conocido como efecto cascada (O'Brien E., 2012). Para evitar estas situaciones no deseadas, es preciso hacer un ajuste adecuado del modelo.

En la actualidad, el modelo HARMONIE-AROME a $1 \mathrm{~km}$ de resolución espacial está siendo utilizado en AEMET para dar apoyo meteorológico y oceanográfico a Puertos del Estado dentro del proyecto SAMOA. Se ejecuta en cuatro dominios espaciales (figura 19).

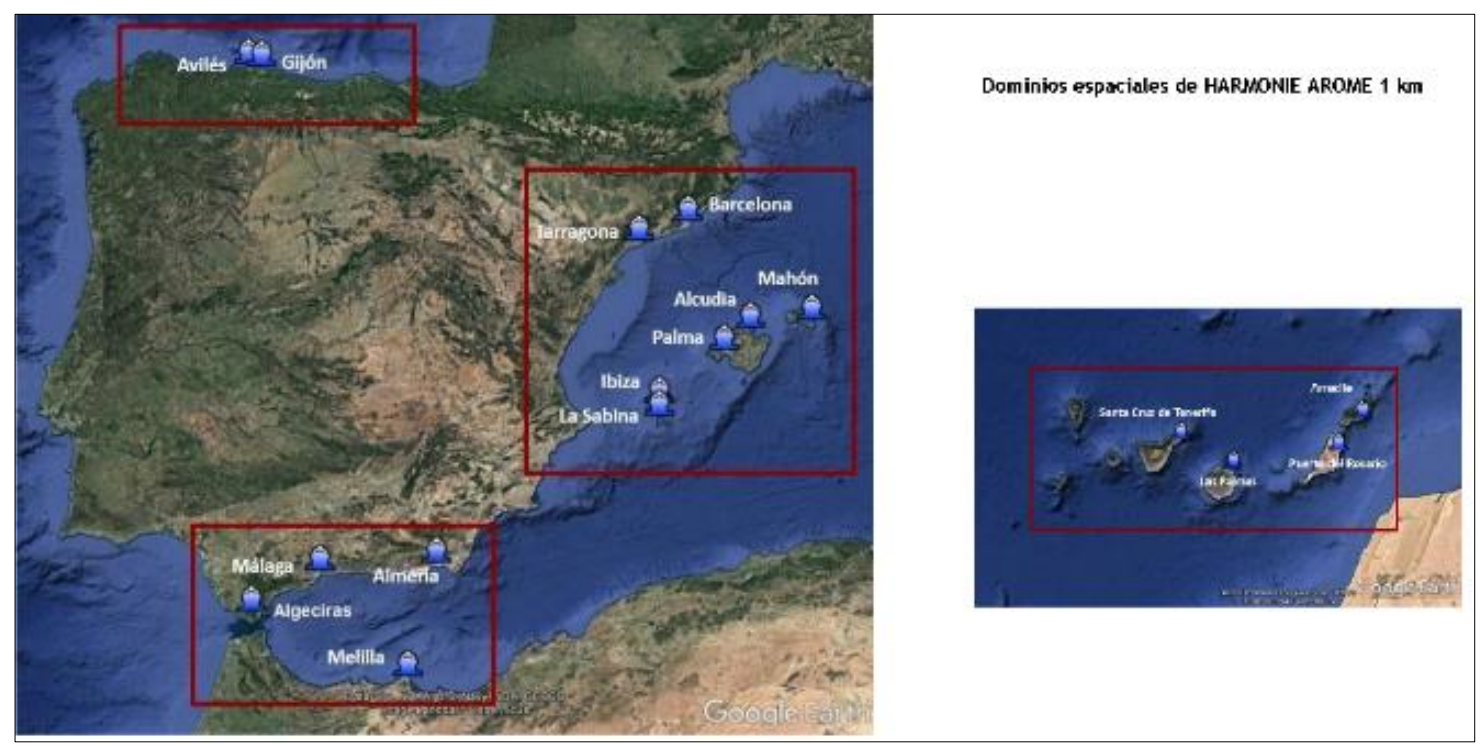

Figura 19. Áreas costeras donde se ejecuta el modelo HARMONIE-AROME a $1 \mathrm{~km}$ de resolución horizontal.

La figura 20 presenta el campo de viento en superficie previsto para el 10-9-2017 en dos alcances concretos (16 UTC y 18 UTC, respectivamente). En ambos, se reconoce plenamente el sistema regional de vientos: Cierzo-Mestral-Tramontana y la línea de convergencia mesoescalar. Ahora bien, el viento difiere del observado, ya que el modelo no vio la entrada del NW en LEBL previa a la incursión del NE. 
La incertidumbre en el posicionamiento de la línea de convergencia puede apreciarse según los distintos alcances previos.

Esta versión del modelo está anidada directamente al modelo IFS del ECMWF. Además, no posee un ciclo de asimilación de observaciones. Estos dos motivos podrían suponer un factor limitante a la hora de pronosticar la posición de la línea de convergencia.
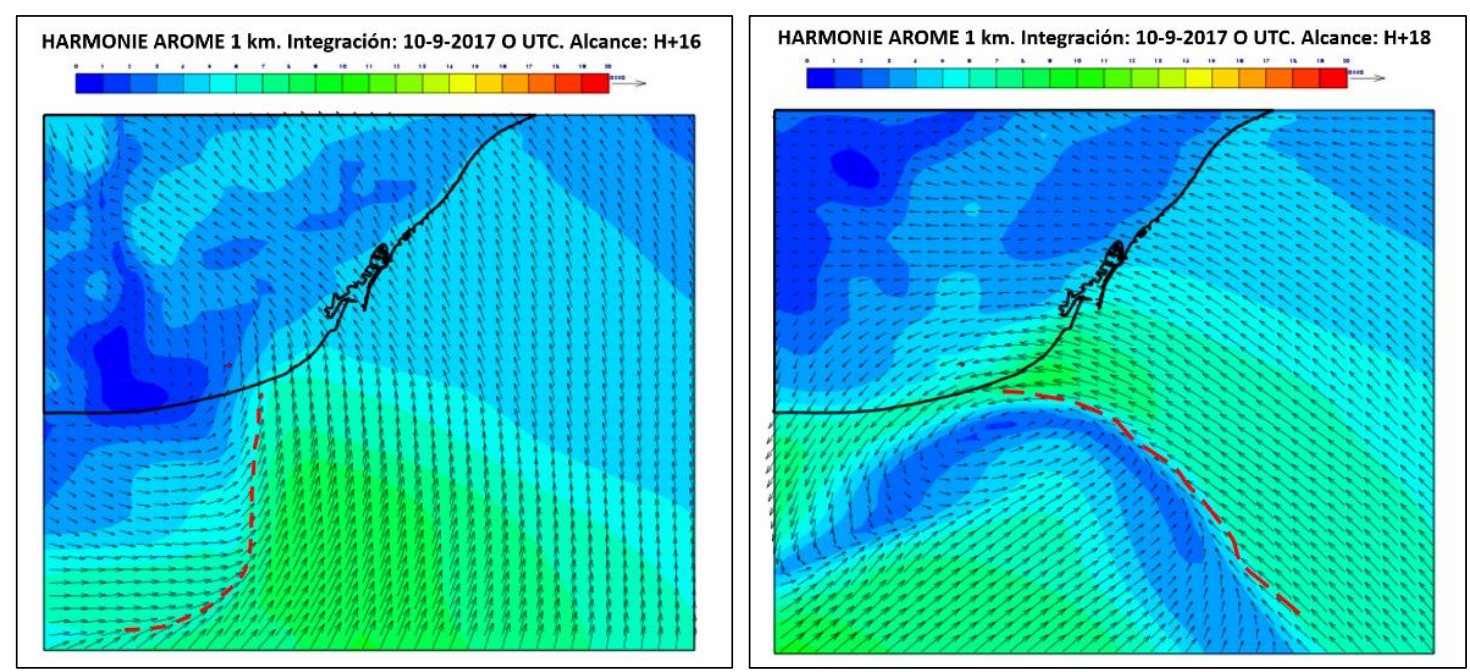

Figura 20.- Viento previsto en superficie por el modelo HARMONIE-AROME a $1 \mathrm{~km}$ de resolución horizontal, en la pasada de las 0 UTC del 10-9-2017 y alcances $\mathrm{H}+16$ y $\mathrm{H}+18$, respectivamente. La línea discontinua roja denota la línea de convergencia. El punto marcado representa LEBL.

\subsection{Modelo de predicción probabilista AEMET-GSREPS}

Las ecuaciones fundamentales que describen el comportamiento de la atmósfera son altamente no lineales, presentando un comportamiento típico de un sistema caótico, motivo por el cual es inviable su resolución analíticamente.

El estado del arte de la predicción no posibilita el pronóstico determinista de la línea de convergencia en el entorno de LEBL. Al tratarse de un cambio súbito del campo viento superficial en un entorno muy local, es necesario recurrir a una interpretación probabilista mediante el uso de un Sistema de Predicción por Conjuntos (en inglés, Ensemble Prediction System, EPS) de corto plazo. Esta visión supone un método plausible de cuantificar la incertidumbre asociada a la predicción del viento (figura 21).

Una buena predicción ha de tener un enfoque dual, entre determinista y probabilista, dando más peso a una u a otra dependiendo de la predecibilidad de la situación que se desea pronosticar.

El modelo AEMET- $\gamma$ SREPS es un sistema multi-modelo (consideración de varios modelos de predicción), multi-análisis (consideración de varios condiciones iniciales) y multicontorno (consideración de varias condiciones de contorno), compuesto por 20 miembros (García-Moya et al., 2007). 
De entre todos los miembros y campos que se predicen, en la figura 22 se ejemplificará con dos concretos. En ambos se representa la temperatura pseudoequivalente y el viento en el nivel de $1000 \mathrm{hPa}$. La discontinuidad horizontal sincronizada que muestran ambos campos permite inferir la línea de convergencia, trazada en amarillo.

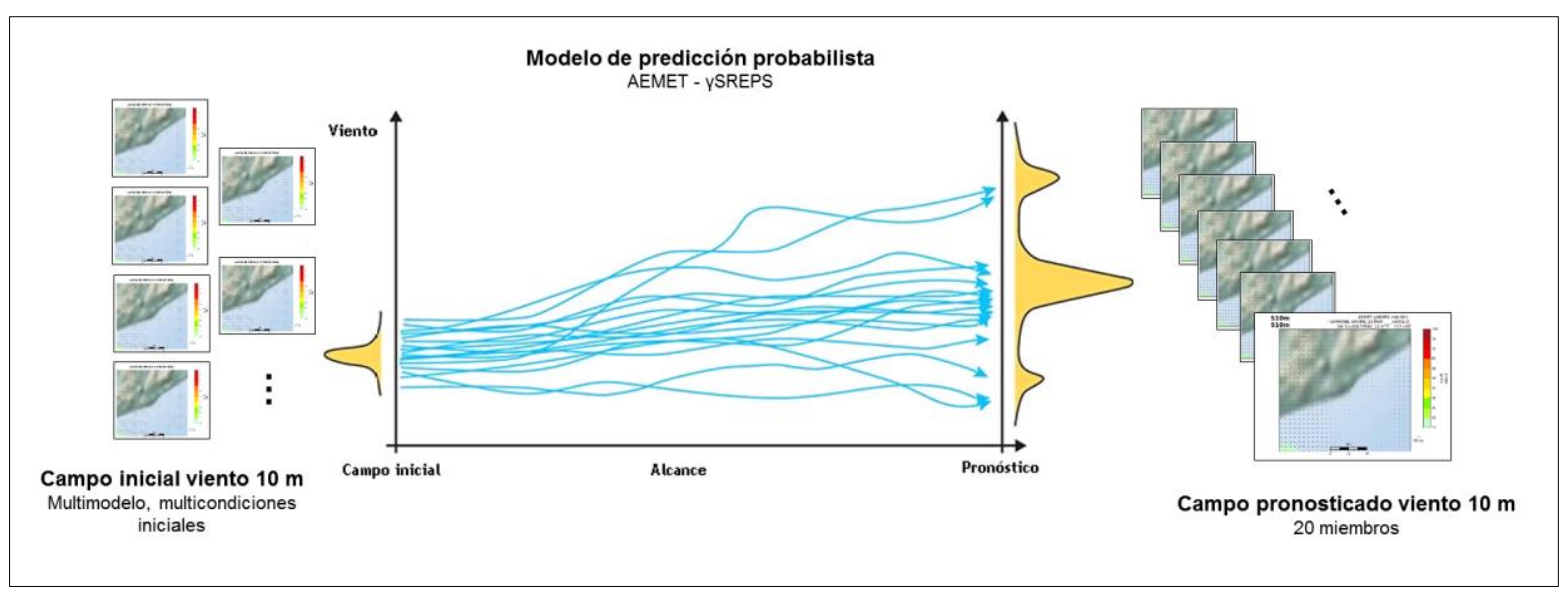

Figura 21. Modelo probabilista AEMET ySREPS: partiendo de varios análisis iniciales del viento a 10 metros en LEBL, se pronostican varios escenarios futuros pesados por una probabilidad. Fuente: adaptado del EPS del ECMWF.

El valor añadido que ofrece una visión probabilista podría resumirse como sigue:

- Hay incertidumbres espaciales y temporales asociadas a la línea de convergencia simulada en el AEMET-үSREPS. La incertidumbre espacial queda bien reflejada en los dos miembros que se muestran en la figura 22.

- Entre 6 y 8 miembros sitúan la convergencia en las inmediaciones del aeropuerto: digamos que la predecibilidad se sitúa alrededor del $30 \%$ y $40 \%$. El AEMET- $\gamma S R E P S$ tenía información de la posibilidad de ocurrencia de un entorno favorable a cizalladura.

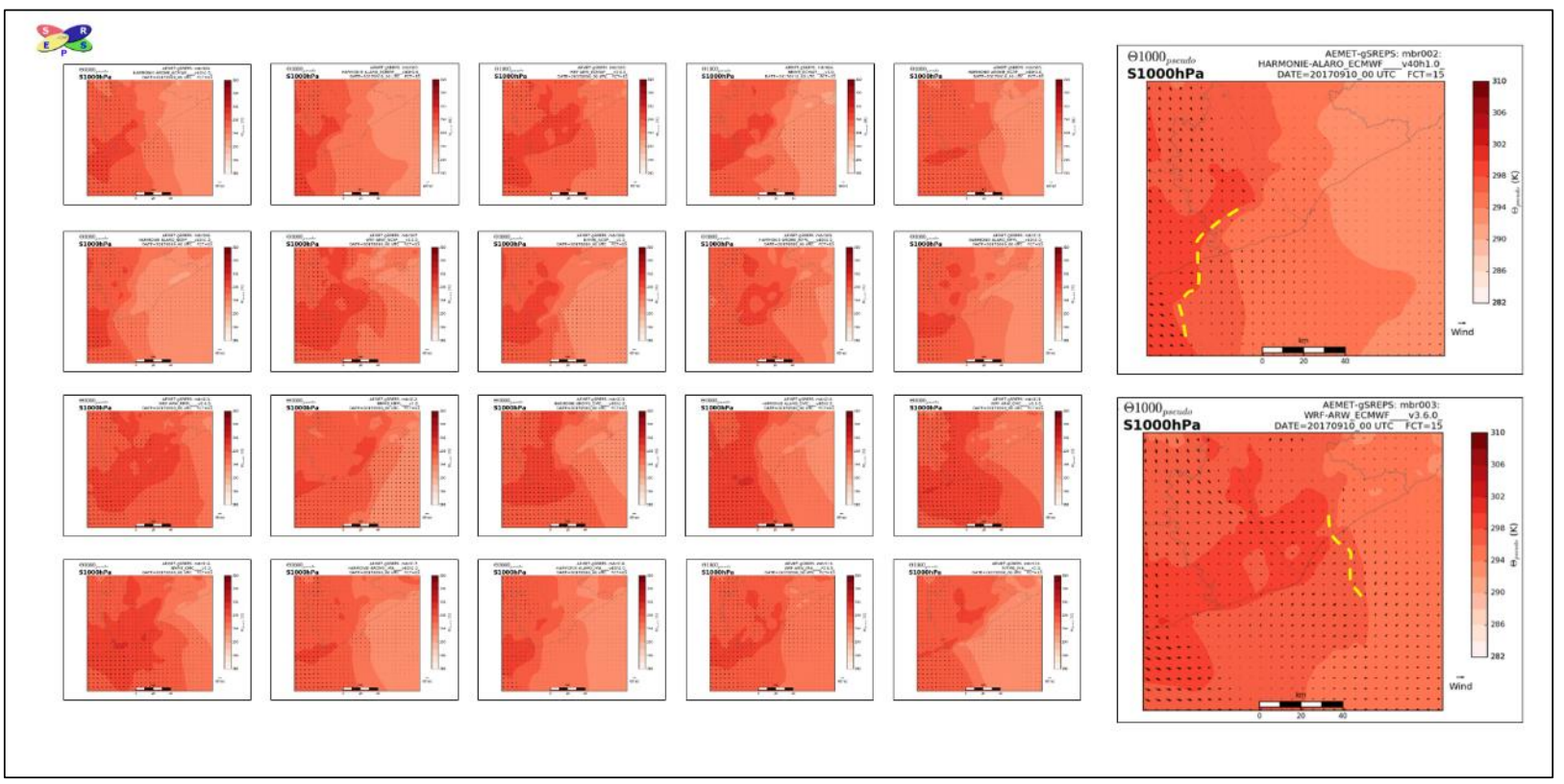


Figura 22. Salidas del campo de temperatura pseudoequivalente y viento en el nivel de $1000 \mathrm{hPa}$ del modelo probabilista AEMET-ySREPS para el 10-9-2017 en el alcance $\mathrm{H}+15$ (a las 15 UTC). Los miembros 2 y 3 (ampliados a la derecha) muestran gráficamente la incertidumbre asociada a la localización de la línea de convergencia.

\subsection{Predicción aeronáutica}

El METAR es un informe meteorológico ordinario en el que se recogen las variables meteorológicas observadas. Por otra parte, el TAF es un informe de pronóstico meteorológico. Ambos se elaboran para un aeródromo y van dirigidos a la navegación aérea.

La tabla 2 muestra, de forma sintetizada, el METAR y TAF válidos para ese día. Se ha optado por utilizar un sencillo código de colores para facilitar el seguimiento de la secuencia temporal de la dirección del viento en superficie a lo largo del día 10-9-2017 en LEBL.

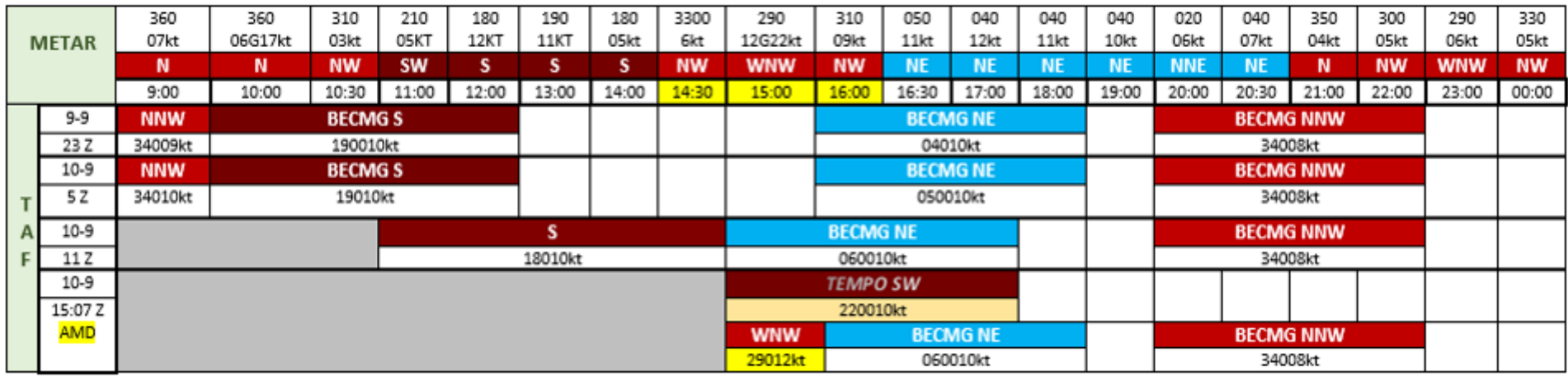

Tabla 2. Viento en los METAR y TAF emitidos el 10-9-2017. Los colores diferencian las direcciones del viento.

En la fila superior, se muestra el viento observado en LEBL que fue reportado en el METAR. La evolución a lo largo de todo el día puede seguirse de izquierda a derecha. Llama la atención la gran variabilidad: a las 11 UTC, el viento era del S-SW, pasando, poco después a NW. Posteriormente roló a un NE, para finalmente acabar en N-NW.

A pesar de no ser un día fácil para elaborar el pronóstico del viento, los TAF emitidos desde el día anterior ya advertían de los diferentes cambios posteriormente observados. Esto puede comprobarse, por simple inspección visual, sobre la tabla 2. Cada discontinuidad en la dirección del viento (cambios de color) se ubica dentro del grupo de cambio del TAF BECMG, el cual significa: «en un cierto intervalo temporal, el viento cambiará a una intensidad y dirección determinada, permaneciendo en ese estado".

El siguiente ejemplo clarifica lo anteriormente comentado:

\begin{tabular}{|c|c|c|c|c|c|c|}
\hline & $68007 \mathrm{kt}$ & $60006 \mathrm{Gl} 17$ & $31003 \mathrm{k}$ & $21005 \mathrm{~K}$ & $18012 \mathrm{KT}$ \\
\hline & & $\mathbf{N}$ & $\mathbf{N}$ & NW & sw & $s$ \\
\hline & METAR & 9:00 & $10: 00$ & $10: 30$ & $11: 00$ & $: 00$ \\
\hline \multirow{2}{*}{ TAF } & 9.9 & NNW & \multicolumn{4}{|c|}{ BECMG $S$} \\
\hline & $\frac{1300}{2}$ & & \\
\hline
\end{tabular}

Cuando el TAF entra en vigor el día 9-9-2017 a las 23 UTC, el viento era de componente N-NW en LEBL, pronosticándose un cambio entre las 10 UTC y las 12 UTC a S. La escala de colores de la fila superior confirma que ese cambio observado (de rojo a granate) queda dentro del grupo de cambio. 
Es importante advertir que el TAF no predijo el cambio de viento de $\mathrm{S}$ a NW que tuvo lugar a las 14:30 UTC. Por este motivo, se emitió una enmienda a las 15:07 UTC.

En efecto, el TAF del día 10-9-2017 a las 11 UTC contemplaba lo siguiente: «partiendo de un viento del S, se prevé que entre las 15 UTC y 17 UTC, el viento rolará a NE, permaneciendo en ese estado». Ahora bien, a las 14:30 UTC se observa viento del WNW, habiéndose previsto del NE. Así, se efectúa la correspondiente enmienda a las 15:07 UTC, que puede leerse en la fila inferior de la tabla 2 como sigue: «a las 15:07 UTC se observa un viento del NW. En algún momento, entre las 16 y 18 , se prevé que éste cambiará a NE».

El cambio no previsto en el TAF comentado anteriormente, va en consonancia con la dispersión que se apreciaba previamente en el meteograma del modelo HARMONIEAROME en LEBL (figura 15). Este hecho evidencia la incertidumbre espacio-temporal asociada a la predicción de cualquier fenómeno de mesoescala, la cual puede cuantificarse haciendo uso de un sistema de predicción por conjuntos (EPS).

En relación con la cizalladura, es importante recordar que en los TAF no se realiza un pronóstico de dicha variable. La información de cizalladura en LEBL puede obtenerse únicamente mediante los sensores de viento de las cabeceras (solo para la cizalladura horizontal que tiene lugar sobre la pista) o mediante las notificaciones de los pilotos a TWR. En este último caso, TWR debiera incluir la información de cizalladura en el mensaje ATIS; una vez incluida en el ATIS, es responsabilidad de la OMA cifrarla en el METAR. En el presente caso llama la atención que, analizados todos los ATIS y METAR del día 10-09-2017, no se comunica cizalladura en ninguno de ellos.

No obstante, también es cierto que los cambios de viento pronosticados en el TAF (con antelación y un grado de acierto elevado) implicaban de por sí situaciones transitorias de cizalladura. El hecho de que la línea de convergencia, tras cruzar las pistas, se quedara durante un cierto tiempo en las proximidades del aeropuerto y de que, al tratarse de un puente festivo el tráfico en LEBL era muy elevado, implicó que el impacto en las operaciones fuera mucho mayor que en otras ocasiones en las que se produjo un fenómeno similar. 


\section{Conclusiones}

A continuación se muestran las cuatro ideas más importantes que subyacen a este estudio:

- Una corriente de densidad mesoescalar atrapada en la costa fue la precursora del establecimiento de un entorno de cizalladura en LEBL el día 10-9-2017, entre las 16 UTC y las 18 UTC. Se trata de un fenómeno conocido, bastante estudiado en otras áreas geográficas y que recientemente se ha observado que es relativamente común en esta zona (González et al., 2018).

- El estado del arte de la predicción no permite un pronóstico estrictamente determinista de un fenómeno mesoescalar, como el que es objeto de este estudio, cuya afectación se circunscribía a un espacio muy limitado y en un reducido periodo de tiempo. Por este motivo, debe tenerse en cuenta el valor añadido que aportan los Sistemas de Predicción por Conjuntos (EPS) mediante la consideración de diversos escenarios.

- Aun tratándose de una situación de baja predecibilidad, el pronóstico de aeródromo fue muy satisfactorio, logrando predecir la mayor parte de los cambios de viento producidos ese día. La inclusión de la enmienda fue síntoma de la vigilancia que se tenía de la situación.

- El hecho de que la línea de cizalladura asociada a la corriente de densidad mesoescalar se quedara durante un cierto tiempo en el entorno del aeropuerto y que éste operaba en condiciones de tráfico elevado dado el carácter festivo de la fecha en cuestión, supuso que el impacto sobre las operaciones fuera mucho mayor que en otros episodios similares. 


\section{Referencias}

Bengtsson, L.; Andrae, U.; Aspelien, T.; Batrak, Y.; Calvo Sánchez, F. J.; Rooy, W.; Gleeson, E.; Hansen Sass, B.; Homileid, M.; Hortal, M.; Ivarsson, K.; Lenderink, G.; Niemelä, S.; Nielsen, K. P.; Onvlee, J.; Rontu, L.; Samuelsson, P.; Santos-Muñoz, D.; Subías Díaz-Blanco, A.; Tijm, A.; Toll, V.; Yang, X.; Ødegaard KØlgzow, M. (2017): The HARMONIE-AROME Model Configuration in the ALADIN-HIRLAM NWP System. American Meteorological Society. Monthly Weather Review. 145(5). 16 pp.

García-Moya, J. A.; Callado-Pallarés, A.; Santos-Burguete, C.; Santos-Muñoz, D.; Simarro-Grande, J. P. (2007): Predicción a mesoescala; Fenómenos severos; Modelos numéricos; Predicción por conjuntos Multimodelo. 2007. Boletín de la Asociación Meteorológica Española. 5(16). 5 pp.

González, Sergi; Callado, Alfons; Werner, Ernest; Escribà, Pau; Bech, Joan (2018): Coastally-Trapped Disturbances caused by the Tramontane wind on the NorthWestern Mediterranean: Numerical study and Sensitivity to Short-wave Radiation. Quarterly Journal of the Royal Meteorological Society. 48 pp.

Markowski, P.; Richardson Y. (2010): Mesoscale Meteorology in Midlatitudes. ISBN: 978-0-470-74213-6. 430 pp.

O'Brien E. (2012): Numerical stability and kinetic energy spectra in high-resolution HARMONIE. HIRLAM Newsletter, No. 59, 6 pp. 


\section{Acrónimos utilizados}

A lo largo de esta nota técnica se han utilizado los siguientes acrónimos y abreviaturas utilizadas por economía de lenguaje y que merecen ser aclaradas:

- ATIS: Automatic Terminal Information Service. Información automática en la que se detallan las condiciones generales de un aeropuerto concreto.

- ECMWF: Centro Europeo de Predicción a Medio Plazo.

- LEBL: es el indicativo OACl, internacionalmente admitido, para referirse al aeropuerto de Barcelona-El Prat.

- METAR: Meteorological Aerodrome Report: observación de aeródromo dirigida a la navegación aérea.

- OMA: Oficina Meteorológica de Aeródromo. En este caso, se está refiriendo a la del aeropuerto de Barcelona- El Prat.

- SPECI: Informe meteorológico especial de aeródromo cuya difusión tiene lugar cuando se cumplen determinados criterios de empeoramiento o mejoría de las condiciones meteorológicas.

- TAF: Terminal Aerodrome Forecast: predicción de aeródromo dirigida a la navegación aérea.

- TWR: Torre de control.

- OACl: Organización de Aviación Civil Internacional.

- UGDD: Unidad de Grabación de Datos Digital.

Hay que advertir que las direcciones del viento se tipifican por su abreviaturas en inglés, por ejemplo: NE: viento del nordeste; NW: viento del noroeste, etc. 


\section{Agradecimientos}

Los autores desean mostrar su agradecimiento al Gestor de Navegación Aérea (ENAIRE) y al personal de la Oficina Meteorológica del aeropuerto de Barcelona-El Prat. 


\section{AFMet \\ Agencia Estatal de Meteorología}

